\title{
CONVEX ALGEBRAIC GEOMETRY OF CURVATURE OPERATORS
}

\author{
RENATO G. BETTIOL, MARIO KUMMER, AND RICARDO A. E. MENDES
}

\begin{abstract}
We study the structure of the set of algebraic curvature operators satisfying a sectional curvature bound under the light of the emerging field of Convex Algebraic Geometry. More precisely, we determine in which dimensions $n$ this convex semialgebraic set is a spectrahedron or a spectrahedral shadow; in particular, for $n \geq 5$, these give new counter-examples to the Helton-Nie Conjecture. Moreover, efficient algorithms are provided if $n=4$ to test membership in such a set. For $n \geq 5$, algorithms using semidefinite programming are obtained from hierarchies of inner approximations by spectrahedral shadows and outer relaxations by spectrahedra.
\end{abstract}

\section{INTRODUCTION}

The emerging field of Convex Algebraic Geometry originates from a natural coalescence of ideas in Convex Geometry, Optimization, and Algebraic Geometry, and has witnessed great progress over the last few years, see [BPT13] for surveys. The main objects considered are convex semialgebraic subsets of vector spaces, such as spectrahedra and their shadows; and their study has led to remarkable achievements in optimization problems for polynomials in several variables. In particular, semidefinite programming on spectrahedral shadows is a far-reaching generalization of linear programming on convex polyhedra, and an area of growing interest due to its numerous and powerful applications, see e.g. [AL12].

The raison d'être of this paper is to shed new light on curvature operators of Riemannian manifolds with sectional curvature bounds from the viewpoint of Convex Algebraic Geometry. More importantly, we hope that the connections established here will serve as foundations for developing further ties between the exciting new frontiers conquered by Convex Algebraic Geometry and classical objects and open problems from Geometric Analysis and Riemannian Geometry.

Recall that a semialgebraic set is a subset $S \subset \mathbb{R}^{n}$ defined by boolean combinations of finitely many polynomial equalities and inequalities; for example, the set $S \subset \mathbb{R}^{4}$ consisting of $(a, b, c, x) \in \mathbb{R}^{4}$ such that $a x^{2}+b x+c=0$ and $a \neq 0$ is a semialgebraic set. The celebrated Tarski-Seidenberg Theorem states that linear projections of semialgebraic sets are also semialgebraic. As an illustration, consider the image $\pi(S) \subset \mathbb{R}^{3}$ of $S \subset \mathbb{R}^{4}$ under the projection $\pi(a, b, c, x)=(a, b, c)$. It consists precisely of $(a, b, c) \in \mathbb{R}^{3}$ with $a \neq 0$ for which

$$
\exists x \in \mathbb{R} \quad \text { such that } a x^{2}+b x+c=0,
$$

Date: February 10, 2021.

2020 Mathematics Subject Classification. 14P10, 53B20, 53C21, 90C22. 
and it can also be described by finitely many polynomial equalities and inequalities (without quantifiers), namely:

$$
b^{2}-4 a c \geq 0
$$

The algorithmic process of rewriting a quantified polynomial sentence, such as (1.1), as an equivalent polynomial sentence without quantifiers, such as (1.2), is known as Quantifier Elimination. This method generalizes the Tarski-Seidenberg Theorem as formulated above, and has deep consequences in Logic, Model Theory, and Theoretical Computer Science.

An elementary application of Quantifier Elimination to Riemannian Geometry is to eliminate the quantifier $\forall$ from the sentence that defines a sectional curvature bound. For example, the condition sec $\geq k$ for an algebraic curvature operator $R: \wedge^{2} \mathbb{R}^{n} \rightarrow \wedge^{2} \mathbb{R}^{n}$, is given by the (quantified) sentence

$$
\forall \sigma \in \operatorname{Gr}_{2}^{+}\left(\mathbb{R}^{n}\right), \quad \sec _{R}(\sigma):=\langle R(\sigma), \sigma\rangle \geq k,
$$

where $\operatorname{Gr}_{2}^{+}\left(\mathbb{R}^{n}\right)=\left\{X \wedge Y \in \wedge^{2} \mathbb{R}^{n}:\|X \wedge Y\|=1\right\}$ is the (oriented) Grassmannian of 2-planes in $\mathbb{R}^{n}$, which is a real algebraic variety hence also a semialgebraic set. This had been observed, among others, by Weinstein [Wei72, p. 260]:

there exist finitely many polynomial inequalities in the $R_{i j k l}$ 's such that, given any curvature tensor, one could determine whether it is positive sectional by evaluating the polynomials and checking whether the results satisfy the inequalities.

In other words, the sets

$$
\mathfrak{R}_{\mathrm{sec} \geq k}(n):=\left\{R \in \operatorname{Sym}_{b}^{2}\left(\wedge^{2} \mathbb{R}^{n}\right): \sec _{R} \geq k\right\}
$$

are semialgebraic. Here, the subscript ${ }_{b}$ indicates that $R \in \operatorname{Sym}^{2}\left(\wedge^{2} \mathbb{R}^{n}\right)$ satisfies the first Bianchi identity, see Section 2 for preliminaries on Riemannian Geometry and curvature operators. The first Bianchi identity is stated in (2.2). We stress that $\Re_{\mathrm{sec} \geq 0}(n)$ can be thought of as the subset of all forms of degree two in the homogeneous coordinate ring of the Grassmannian $\mathrm{Gr}_{2}(n)$ that are nonnegative on the real part of $\mathrm{Gr}_{2}(n)$. This point of view might be helpful for readers with a background in Convex Algebraic Geometry. Weinstein [Wei72, p. 260] continues:

It would be useful to know these inequalities explicitly. [...] Unfortunately, the [Quantifier Elimination] procedure is too long to be used in practice even with the aid of a computer.

Despite all technological advances, this remains true today, almost 50 years later. Although such an explicit description of $\Re_{\sec \geq k}(n)$ is still elusive, in this paper we provide new information about these semialgebraic sets. Besides being of intrinsic interest, we expect this will lead to new global results in differential geometry.

A fundamental example of convex semialgebraic set is the cone $\left\{A \in \operatorname{Sym}^{2}\left(\mathbb{R}^{d}\right)\right.$ : $A \succeq 0\}$ of positive-semidefinite matrices. Preimages of this cone under affine maps $\mathbb{R}^{n} \rightarrow \operatorname{Sym}^{2}\left(\mathbb{R}^{d}\right)$ are also convex semialgebraic, and are called spectrahedra. They generalize polyhedra, which correspond to affine maps with image in the subspace of diagonal matrices. In contrast to polyhedra, the linear projection of a spectrahedron may fail to be a spectrahedron. Nevertheless, these projections are convex semialgebraic sets, and are called spectrahedral shadows. Following a question of Nemirovski [Nem07] in his 2006 ICM plenary address, Helton and Nie [HN09, p. 790] conjectured that every convex semialgebraic set is a spectrahedral shadow. 
Remarkably, this turned out not to be the case, as very recently discovered by Scheiderer [Sch18b]. Further counter-examples were subsequently found in [Faw19]. We describe a convenient and digestible criterion, which follows from Scheiderer's work [Sch18b], for the cone of nonnegative polynomials inside a given vector space of polynomials not to be a spectrahedral shadow that can be of independent interest.

Our first main result describes how sets of algebraic curvature operators with sectional curvature bounds fit in the above taxonomy of convex semialgebraic sets, providing yet another class of counter-examples to the Helton-Nie Conjecture:

Theorem A. For all $k \in \mathbb{R}$, each of the sets $\mathfrak{R}_{\mathrm{sec} \geq k}(n)$ and $\mathfrak{R}_{\sec \leq k}(n)$ is:

(1) not a spectrahedral shadow, if $n \geq 5$;

(2) a spectrahedral shadow, but not a spectrahedron, if $n=4$;

(3) a spectrahedron, if $n \leq 3$.

We state Theorem A in the above manner for the sake of completeness, despite the fact that some claims were previously known. More precisely, statement (3) follows trivially from the equivalence, in dimensions $n \leq 3$, between $\sec _{R} \geq k$ and $R-k$ Id $\succeq 0$; analogously for sec $\leq k$ (which we omit henceforth, see Remark 2.2). Furthermore, the first part of statement (2) follows from the so-called Thorpe's trick [Tho72], see Proposition 3.1; namely, the equivalence, in dimension $n=4$, between $\sec _{R} \geq k$ and the existence of $x \in \mathbb{R}$ such that $R-k \operatorname{Id}+x * \succeq 0$, where $*$ is the Hodge star operator. In other words, $\mathfrak{R}_{\mathrm{sec} \geq k}(4)$ is the image of the spectrahedron $\left\{R \in \operatorname{Sym}^{2}\left(\wedge^{2} \mathbb{R}^{4}\right): R-k\right.$ Id $\left.\succeq 0\right\}$ under the orthogonal projection $\operatorname{Sym}^{2}\left(\wedge^{2} \mathbb{R}^{4}\right) \rightarrow \operatorname{Sym}_{b}^{2}\left(\wedge^{2} \mathbb{R}^{4}\right)$, whose kernel is spanned by $*$. We point out that Thorpe's trick is actually an instance of a much older result due to Finsler [Fin36], see Lemma 2.10; a fact that seems to have gone unnoticed thus far.

For readers interested in strict sectional curvature bounds $\sec >k$ and $\sec <k$, we note that statements (1) and (2) in Theorem A carry over to this setting, see Remarks 2.2 and 2.4. However, in keeping with the Convex Algebraic Geometry literature, all spectrahedra are (by definition) closed sets. Thus, $\mathfrak{R}_{\sec >k}(n)$ and $\mathfrak{R}_{\text {sec }<k}(n), n \leq 3$, are, strictly speaking, not spectrahedra. Of course, this is just a matter of convention, and sec $>k$ is clearly equivalent to $R-k$ Id $\succ 0$ if $n \leq 3$.

Although $\mathfrak{R}_{\mathrm{sec} \geq k}(n), n \geq 5$, fails to be a spectrahedral shadow, our second main result provides natural approximations by spectrahedral shadows and spectrahedra:

TheOREM B. For all $k \in \mathbb{R}$ and $n \geq 2$, there are inner and outer approximations of $\mathfrak{R}_{\mathrm{sec} \geq k}(n)$ by nested sequences $\mathfrak{I}_{m}$ of spectrahedral shadows and $\mathfrak{O}_{m}$ of spectrahedra, $\mathfrak{I}_{0} \subset \mathfrak{I}_{1} \subset \cdots \subset \mathfrak{I}_{m} \subset \cdots \subset \mathfrak{R}_{\mathrm{sec} \geq k}(n) \subset \cdots \subset \mathfrak{O}_{m} \subset \cdots \subset \mathfrak{O}_{1} \subset \mathfrak{O}_{0}, \quad m \geq 0$, which are $\mathrm{O}(n)$-invariant and satisfy $\overline{\bigcup_{m \geq 0} \mathfrak{I}_{m}}=\mathfrak{R}_{\mathrm{sec} \geq k}(n)=\bigcap_{m \geq 0} \mathfrak{O}_{m}$.

The inner approximation by spectrahedral shadows $\mathfrak{I}_{m}, m \geq 0$, is constructed in the same spirit as the Lasserre hierarchy [Las01, Par00]; and, if $k=0$, the first step $\mathfrak{I}_{0}$ coincides precisely with the subset of curvature operators with strongly nonnegative curvature, see [BM17, BM18]. The outer approximation by spectrahedra $\mathfrak{O}_{m}$, $m \geq 0$, is given by curvature operators with positive-semidefinite curvature terms in all Weitzenböck formulae for traceless symmetric $p$-tensors with $p \leq m+1$, see [BM, Thm. A]; and, if $k=0$, the first step $\mathfrak{O}_{0}$ coincides with the subset of curvature operators with nonnegative Ricci curvature. Since all $\mathfrak{I}_{m}$ and $\mathfrak{O}_{m}$ are $\mathrm{O}(n)$-invariant, these approximations are geometric, in the sense that they define coordinate-free curvature conditions. We remark that $\mathfrak{I}_{0}=\mathfrak{R}_{\mathrm{sec} \geq k}(n)$ if and only if $n \leq 4$, while 
$\mathfrak{O}_{0}=\mathfrak{R}_{\mathrm{sec} \geq k}(n)$ if and only if $n=2$. By Theorem A, these approximations do not stabilize after finitely many steps $m \geq 0$, for all $n \geq 5$.

In our third main result, we restrict to dimension $n=4$ to exploit the description of $\Re_{\mathrm{sec} \geq k}(4)$ as a spectrahedral shadow in order to obtain an explicit description of this set as an algebraic interior, see Definition 2.5.

TheOREM C. The set $\mathfrak{R}_{\mathrm{sec} \geq k}(4)$ is an algebraic interior with minimal defining polynomial $\mathfrak{p}_{k}: \operatorname{Sym}_{b}^{2}\left(\wedge^{2} \mathbb{R}^{4}\right) \rightarrow \mathbb{R}$, given by

$$
\mathfrak{p}_{k}(R)=\operatorname{disc}_{x}(\operatorname{det}(R-k \operatorname{Id}+x *)) .
$$

More precisely, $\mathfrak{R}_{\mathrm{sec} \geq k}(4)=\overline{\mathcal{C}_{k}}$, where $\mathcal{C}_{k}$ is the only connected component of the set $\left\{R \in \operatorname{Sym}_{b}^{2}\left(\wedge^{2} \mathbb{R}^{4}\right): \mathfrak{p}_{k}(R)>0\right\}$ such that $(k+1) \operatorname{Id} \in \overline{\mathcal{C}_{k}}$.

In the above, $\operatorname{disc}_{x}$ denotes the discriminant in $x$, see Subsection 2.3. Using a complexification trick (3.3), the above polynomial $\mathfrak{p}_{k}(R)$ can be seen as the discriminant of a symmetric matrix, and hence more efficiently computed, e.g. using [Par02]. A result related to Theorem $\mathrm{C}$, where (1.3) is considered as a polynomial in $k \in \mathbb{R}$, was recently obtained by Fodor [Fod20].

Although Theorem C falls short of giving a description of $\Re_{\mathrm{sec} \geq k}(4)$ as a semialgebraic set, it provides an explicit such description of another semialgebraic set that has $\mathfrak{R}_{\mathrm{sec} \geq k}(4)$ as (the closure of) one of its connected components. Moreover, it follows from Theorem $\mathrm{C}$ that the algebraic boundary of $\mathfrak{R}_{\mathrm{sec} \geq k}(4)$, i.e., the Zariski closure of its topological boundary, is the zero set of the polynomial (1.3).

As a computational application of the description of $\Re_{\sec \geq k}(4)$ as a spectrahedral shadow, we provide an efficient algorithm (different from semidefinite programming) to determine when a given $R \in \operatorname{Sym}_{b}^{2}\left(\wedge^{2} \mathbb{R}^{4}\right)$ belongs to this set. This algorithm is based on Sturm's root counting method, and detects membership in $\mathfrak{R}_{\mathrm{sec} \geq 0}(4)$ and also in $\Re_{\sec >0}(4)$, see Algorithms 2 and 3; the cases of other sectional curvature bounds (strict or not) are easily obtained from these with obvious modifications. For $n \geq 5$, the approximations given by Theorem $\mathrm{B}$ allow to use an iteration of semidefinite programs (see Algorithm 1) to detect membership in $\mathfrak{R}_{\mathrm{sec} \geq k}(n)$ except for a set of measure zero of bad inputs, where the algorithm does not halt.

Straightforward generalizations of Theorems A, B, and C to sets of algebraic curvature operators on vectors spaces with semi-Riemannian inner products, satisfying a suitable replacement for $\sec \geq k$, are discussed in Appendix A.

Relevance for applications. Next, we highlight some applications to which this work is pertinent in Optimization, Information Theory, and Data Science.

Semidefinite programming. Semidefinite programming is a generalization of linear programming which attracted a lot of interest, as one can solve under mild assumptions a semidefinite program up to a fixed precision in time that is polynomial in the program description size [NN94]. Typical applications include polynomial optimization [PS03] or combinatorial optimization, e.g. the Max-Cut Problem [Goe98]. The standard form for a semidefinite program (SDP) is:

$$
\min _{x} c_{1} x_{1}+\ldots+c_{n} x_{n}
$$

under the constraint that the real symmetric matrix $A_{0}+x_{1} A_{1}+\ldots+x_{n} A_{n}$ is positive-semidefinite, where $c_{i} \in \mathbb{R}$ and $A_{0}, \ldots, A_{n}$ are real symmetric matrices. Thus the feasible region of an SDP is a spectrahedron. By introducing slack variables one can also run an SDP on spectrahedral shadows. Therefore, in the theory 
of semidefinite programming, it is crucial to find a characterization of this special class of convex sets [Nem07]. While it is easy to see that every spectrahedral shadow is convex and semialgebraic, the first examples of convex semialgebraic sets that are not spectrahedral shadows were only found recently by Scheiderer [Sch18b]. In this work, we provide further such examples (Theorem A). Moreover, we believe that our simplification and concretization of Scheiderer's criterion for being a spectrahedral shadow (Theorem 2.14) will be useful in the future for assessing the applicability of SDP. The question on the expressive power of semidefinite programming is also of ample interest in Theoretical Computer Science because of its intimate connection to the Unique Games Conjecture [Rag08].

Information Geometry. Consider the space of parameters $M$ of a probability distribution $p(x, \theta)$ with the Riemannian metric given by the Fisher information matrix

$$
\mathrm{g}_{a b}(\theta)=\mathrm{E}\left[\frac{\partial^{2} i(x, \theta)}{\partial \theta_{a} \partial \theta_{b}}\right]=\int_{X} \frac{\partial^{2} i(x, \theta)}{\partial \theta_{a} \partial \theta_{b}} p(x, \theta) \mathrm{d} x,
$$

where $\theta=\left(\theta_{1}, \ldots, \theta_{n}\right) \in M$ are local coordinates, $i(x, \theta)=-\log p(x, \theta)$ is the Shannon information, and $x$ is drawn from the value space of the random variable $X$. Such Riemannian manifolds $(M, \mathrm{~g})$ are called statistical manifolds, and are the central objects of study in Information Geometry, which leverages methods from Differential Geometry and Geometric Analysis in Probability Theory, Statistical Inference, and Information Science. Applications of Information Geometry are fast growing and widespread, ranging from neural networks, machine learning and signal processing [Ama16] to complexity of composite systems, evolutionary dynamics, and Monte Carlo stochastic sampling [AJLS17]. Since the Riemannian curvature of the Fisher metric (1.4) allows to detect critical parameter values where a phase transition occurs [AJLS17, p. 100], our Algorithms 1, 2, and 3, in Section 5, may provide valuable computational tools for such applications. Namely, given a probability distribution with $n$ parameters, the full range of sectional curvatures at a point $\theta$ in the corresponding $n$-dimensional statistical manifold $(M, \mathrm{~g})$ can be determined if $n \leq 4$, or approximated if $n \geq 5$, by repeatedly invoking the above algorithms with the curvature operator of $(M, \mathrm{~g})$ at $\theta$ as input, and raising/lowering the corresponding lower/upper curvature bounds.

Geometric Data Analysis. Many tools in Data Science, such as the notion of mean (or centroid), and its use, e.g., in $k$-means clustering, and Principal Component Analysis (PCA), which is fundamental to address the "curse of dimensionality", were originally conceived for data lying inside Euclidean space $\mathbb{R}^{n}$. Given the recent considerable availability and interest in manifold-valued data, the development of suitable nonlinear replacements for these tools became of great importance. Fréchet mean (FM) and Principal Geodesic Analysis (PGA), respectively, are convenient generalizations that attracted particular interest for applications in medical imaging [FCPS04, BJV17]. However, these can only be used assuming that the (known) manifold $(M, \mathrm{~g})$, where the data points lie, satisfies certain geometric constraints. For instance, uniqueness of FM for data points in $(M, \mathrm{~g})$ with sec $\leq K, K>0$, is guaranteed if these points lie inside a geodesic ball of radius $r<\frac{1}{2} \min \left\{\operatorname{inj} M, \frac{\pi}{\sqrt{K}}\right\}$, where inj $M$ is the injectivity radius of $(M, \mathrm{~g})$, see [Afs11]. Similarly, PGA relies on closest-point projection operators onto geodesic submanifolds of $(M, \mathrm{~g})$, which can also be controlled in terms of sectional curvature bounds. Thus, Algorithms 1, 2, 
and 3, in Section 5, through their estimation of sectional curvature bounds, allow to estimate on which (portions of) manifolds FM and PGA can be applied reliably for data analysis. Note that these algorithms only determine sectional curvature bounds point-by-point, so the above strategy involves using a sufficiently dense net of sample points on $(M, \mathrm{~g})$ to which such algorithm is applied.

Discussion of proofs. In order to simplify the exposition, now and throughout the paper we only consider the sectional curvature bound $\mathrm{sec} \geq 0$, for the reasons laid out in Remark 2.2. The proof of Theorem A (1) uses much heavier theoretical machinery than Theorem C, namely the deep recent results of Scheiderer [Sch18b], while Theorem A (2) is a consequence of Theorem C. Theorem B relies on [BM] to produce the outer approximation by spectrahedra, and on an adaptation of the Lasserre hierarchy method for the inner approximation by spectrahedral shadows.

There are three main steps in the proof of Theorem $\mathrm{C}$, which is presented in Section 3. First, a complexification trick (3.3) is used to establish that $\mathfrak{p}:=\mathfrak{p}_{0}$, see (1.3), vanishes on the topological boundary of $\mathfrak{R}_{\mathrm{sec} \geq 0}(4)$, see Proposition 3.2. Second, we show that the vanishing locus of $\mathfrak{p}$ does not disconnect the interior of $\mathfrak{R}_{\mathrm{sec} \geq 0}(4)$ for dimensional reasons, see Proposition 3.5. These two facts already imply that $\mathfrak{R}_{\mathrm{sec} \geq 0}(4)$ is an algebraic interior with defining polynomial $\mathfrak{p}$, so it only remains to prove that $\mathfrak{p}$ is minimal. This follows from irreducibility of $\mathfrak{p}$, which is an application of canonical forms for complex symmetric matrices, see Appendix B.

The intimate connection between algebraic curvature operators and quadratic forms on the Grassmannian of 2-planes is at the foundation of the proof of Theorem A (1). More generally, given a real projective variety $X \subset \mathbb{C} P^{N}$, and a (real) quadratic form $f$ on $X$, that is, an element in the degree 2 part $\mathbb{R}[X]_{2}$ of its homogeneous coordinate ring, the value of $f$ at a real point $x \in X(\mathbb{R})$ is not well-defined, however its sign is. Indeed, $f(\lambda \tilde{x})=\lambda^{2} f(\tilde{x})$ for any representative $\tilde{x} \in \mathbb{R}^{N+1}$ and $\lambda \in \mathbb{R} \backslash\{0\}$. Thus, one may consider the set $\mathrm{P}_{X} \subset \mathbb{R}[X]_{2}$ of all nonnegative quadratic forms on $X(\mathbb{R})$, see (2.9), which clearly contains the set $\Sigma_{X}$ of quadratic forms that are sums of squares of elements in $\mathbb{R}[X]_{1}$, see (2.8). The characterization of varieties $X$ for which $\mathrm{P}_{X}=\Sigma_{X}$ as those of minimal degree is a landmark result recently obtained by Blekherman, Smith, and Velasco [BSV16]. This fits into the broader question of which nonnegative functions are sums of squares, which has a long history, dating back to Minkowski, Hilbert [Hil88] and Artin [Art27].

In the case of the Grassmannian $X=\mathrm{Gr}_{2}(n)$, which is determined by quadratic equations $\omega_{i}=0$ called Plücker relations, see (2.5), the connection alluded to above takes the form of the identification

$$
\mathbb{R}\left[\operatorname{Gr}_{2}(n)\right]_{2} \cong \operatorname{Sym}_{b}^{2}\left(\wedge^{2} \mathbb{R}^{n}\right) .
$$

Namely, $\operatorname{Sym}^{2}\left(\wedge^{2} \mathbb{R}^{n}\right)$ can be identified as usual with quadratic forms on $\wedge^{2} \mathbb{R}^{n}$ by associating each $R$ to $q_{R}(\alpha)=\langle R(\alpha), \alpha\rangle$. On the one hand, $\mathbb{R}\left[\operatorname{Gr}_{2}(n)\right]_{2}$ is by definition the quotient $\operatorname{Sym}^{2}\left(\wedge^{2} \mathbb{R}^{n}\right) / \operatorname{span}\left(\omega_{i}\right)$, since $\omega_{i}$ generate the vanishing ideal of $\operatorname{Gr}_{2}(n)$. On the other hand, the orthogonal complement of $\operatorname{span}\left(\omega_{i}\right)$ in $\operatorname{Sym}^{2}\left(\wedge^{2} \mathbb{R}^{n}\right)$ is exactly $\operatorname{Sym}_{b}^{2}\left(\wedge^{2} \mathbb{R}^{n}\right)$, yielding (1.5), see Subsection 2.2 for details.

Under the identification (1.5), the set $\mathrm{P}_{\mathrm{Gr}_{2}(n)} \subset \mathbb{R}\left[\mathrm{Gr}_{2}(n)\right]_{2}$ corresponds to $\mathfrak{R}_{\mathrm{sec} \geq 0}(n) \subset \operatorname{Sym}_{b}^{2}\left(\wedge^{2} \mathbb{R}^{n}\right)$, while $\Sigma_{\mathrm{Gr}_{2}(n)}$ corresponds to the set of curvature operators with strongly nonnegative curvature, see Example 2.11. As the Grassmannian $\mathrm{Gr}_{2}(4)$ has minimal degree, $\mathrm{P}_{\mathrm{Gr}_{2}(4)}=\Sigma_{\mathrm{Gr}_{2}(4)}$ by [BSV16]. This recovers the Finsler-Thorpe trick, since the only Plücker relation in dimension $n=4$ is given 
by $\omega_{1}(R)=\langle * R, R\rangle=0$. Furthermore, as $\mathrm{Gr}_{2}(n)$ does not have minimal degree for all $n \geq 5$, there exist $P \in \mathrm{P}_{\mathrm{Gr}_{2}(n)} \backslash \Sigma_{\mathrm{Gr}_{2}(n)}$ which translates to the failure of higherdimensional analogues of the Finsler-Thorpe trick (explicit $P$ 's were obtained by Zoltek [Zol79]). Such a $P$ is the key input to apply a criterion of Scheiderer [Sch18b] to show that $\mathrm{P}_{\mathrm{Gr}_{2}(n)} \cong \mathfrak{R}_{\mathrm{sec} \geq 0}(n)$ is not a spectrahedral shadow, as claimed in Theorem A (1). In fact, we extract from [Sch18b] an easily applicable criterion, Theorem 2.14, which implies [Faw19, Thm. 3] and is of independent interest.

More generally, the Grassmannians $\mathrm{Gr}_{k}(n)$ of $k$-planes do not have minimal degree if and only if $2 \leq k \leq n-2$ and $n \geq 5$, and hence $\mathrm{P}_{\mathrm{G} r_{k}(n)} \neq \Sigma_{\mathrm{G} r_{k}(n)}$ in this range. Scheiderer's criterion still applies in this situation, and leads to the conclusion that $\mathrm{P}_{\mathrm{G} r_{k}(n)}, 2 \leq k \leq n-2, n \geq 5$, are not spectrahedral shadows, see Corollary 4.2. Since $\Sigma_{X}$ is a spectrahedral shadow for any projective variety $X$, this can be interpreted as a strengthening of [BSV16] for the class of Grassmannians $X=\operatorname{Gr}_{k}(n) \subset \mathbb{C} P^{\left(\begin{array}{l}n \\ k\end{array}\right)-1}$; namely, $\mathrm{P}_{X}$ is a spectrahedral shadow if and only if $\mathrm{P}_{X}=$ $\Sigma_{X}$. The same strengthening was observed by Scheiderer [Sch18b, Cor. 4.25] for the class of degree $d$ Veronese embeddings $X=\mathbb{C} P^{n} \subset \mathbb{C} P\left({ }^{(n+d}\right)-1$. Nevertheless, such a strengthening does not hold in full generality, as exemplified by curves $X$ of positive genus (e.g., elliptic curves). Such $X$ do not have embeddings of minimal degree, however $\mathrm{P}_{X}$ is always a spectrahedral shadow since it is the dual of the convex hull of a curve, which is a spectrahedral shadow by Scheiderer [Sch18a].

Acknowledgements. It is a great pleasure to thank Bernd Sturmfels for introducing the second-named author to the first- and third-named authors, and suggesting the complexification trick (3.3). We also thank Alexander Lytchak for supporting a visit by the second-named author to the University of Cologne, during which excellent working conditions allowed us to finalize this paper. The first-named author is grateful to the Max Planck Institut für Mathematik in Bonn for the hospitality in the summer of 2019, which made it possible to regularly meet the other authors.

The first-named author was supported by the National Science Foundation grant DMS-1904342, and by the Max Planck Institut für Mathematik in Bonn, and the third-named author was supported by the National Science Foundation grant DMS2005373 and the Deutsche Forschungsgemeinschaft grants DFG ME 4801/1-1 and DFG SFB TRR 191.

\section{Preliminaries}

2.1. Riemannian Geometry. Given a Riemannian manifold $(M, \mathrm{~g})$ and $p \in M$, the curvature operator at $p$ is the symmetric endomorphism $R \in \operatorname{Sym}^{2}\left(\wedge^{2} T_{p} M\right)$,

$$
\langle R(X \wedge Y), Z \wedge W\rangle=\mathrm{g}\left(\nabla_{Y} \nabla_{X} Z-\nabla_{X} \nabla_{Y} Z+\nabla_{[X, Y]} Z, W\right),
$$

where $\nabla$ denotes the Levi-Civita connection, and $\langle\cdot, \cdot\rangle$ denotes the inner product induced by g on $\wedge^{2} T_{p} M$. Curvature operators $R$ satisfy the (first) Bianchi identity:

$$
\langle R(X \wedge Y), Z \wedge W\rangle+\langle R(Y \wedge Z), X \wedge W\rangle+\langle R(Z \wedge X), Y \wedge W\rangle=0,
$$

for all $X, Y, Z, W \in T_{p} M$. Given $X, Y \in T_{p} M$ two g-orthonormal tangent vectors, the sectional curvature of the plane $\sigma$ spanned by $X$ and $Y$ is

$$
\sec (\sigma)=\langle R(X \wedge Y), X \wedge Y\rangle .
$$


Since the present paper is only concerned with pointwise properties of curvature operators, we henceforth identify $T_{p} M \cong \mathbb{R}^{n}$ and define (algebraic) curvature operators as elements $R \in \operatorname{Sym}^{2}\left(\wedge^{2} \mathbb{R}^{n}\right)$ that satisfy the Bianchi identity (2.2). We denote by $\operatorname{Sym}_{b}^{2}\left(\wedge^{2} \mathbb{R}^{n}\right) \subset \operatorname{Sym}^{2}\left(\wedge^{2} \mathbb{R}^{n}\right)$ the subspace of such curvature operators, and by $\mathfrak{b}$ the orthogonal projection onto its complement, so that $\operatorname{Sym}_{b}^{2}\left(\wedge^{2} \mathbb{R}^{n}\right)=\operatorname{ker} \mathfrak{b}$. Elements $R \in \operatorname{Sym}^{2}\left(\wedge^{2} \mathbb{R}^{n}\right)$ are sometimes called modified curvature operators.

We identify $\wedge^{4} \mathbb{R}^{n}$ with a subspace of $\operatorname{Sym}^{2}\left(\wedge^{2} \mathbb{R}^{n}\right)$ via

$$
\langle\omega(\alpha), \beta\rangle=\langle\omega, \alpha \wedge \beta\rangle, \quad \text { for all } \omega \in \wedge^{4} \mathbb{R}^{n}, \alpha, \beta \in \wedge^{2} \mathbb{R}^{n} .
$$

Note that $\wedge^{4} \mathbb{R}^{n}$ is the image of $\mathfrak{b}$, i.e., the orthogonal complement of $\operatorname{Sym}_{b}^{2}\left(\wedge^{2} \mathbb{R}^{n}\right)$.

2.2. Grassmannians and curvature operators. The above classical definitions from Riemannian geometry can be conveniently reinterpreted in terms of the algebraic geometry of the Grassmannian of 2-planes. This relationship forms the raison d'être of this paper.

The natural coordinates $x_{i j}, 1 \leq i<j \leq n$, in $\wedge^{2} \mathbb{C}^{n}$ induced from the standard basis $e_{1}, \ldots, e_{n}$ of $\mathbb{C}^{n}$ are called Plücker coordinates. The Grassmannian

$$
\mathrm{Gr}_{2}(n) \subset \mathbb{P}\left(\wedge^{2} \mathbb{C}^{n}\right) \cong \mathbb{C} P^{\left(\begin{array}{l}
n \\
2
\end{array}\right)-1}
$$

of 2-planes in $\mathbb{C}^{n}$ is the real projective variety defined by the Plücker relations; namely the zero locus of the quadratic forms associated to a basis of $\wedge^{4} \mathbb{R}^{n}$, considered as $\left(\begin{array}{l}n \\ 4\end{array}\right)$ homogeneous quadratic polynomials on (the Plücker coordinates of) $\wedge^{2} \mathbb{C}^{n}$, cf. (2.4). These quadratic forms generate the homogeneous vanishing ideal $I_{\mathrm{Gr}_{2}(n)} \subset \mathbb{C}\left[x_{i j}\right]$ of $\mathrm{Gr}_{2}(n)$, and the homogeneous coordinate ring $\mathbb{C}\left[\mathrm{Gr}_{2}(n)\right]$ of $\operatorname{Gr}_{2}(n)$ is given by $\mathbb{C}\left[x_{i j}\right] / I_{\mathrm{Gr}_{2}(n)}$. The rings $\mathbb{C}\left[x_{i j}\right]$ and $\mathbb{C}\left[\mathrm{Gr}_{2}(n)\right]$, as well as the ideal $I_{\mathrm{Gr}_{2}(n)}$, have natural graded structures; as usual, we denote their degree $d$ part with the subscript $d$.

The Grassmannian of 2-planes in $\mathbb{R}^{n}$ is the set $\mathrm{Gr}_{2}(n)(\mathbb{R})$ of real points of $\mathrm{Gr}_{2}(n)$, which we also denote $\mathrm{Gr}_{2}\left(\mathbb{R}^{n}\right)$. The (oriented) Grassmannian $\mathrm{Gr}_{2}^{+}\left(\mathbb{R}^{n}\right) \subset S^{\left(\begin{array}{c}n \\ 2\end{array}\right)-1}$ in the Introduction is the double-cover of $\mathrm{Gr}_{2}\left(\mathbb{R}^{n}\right) \subset \mathbb{R} P^{\left(\begin{array}{c}n \\ 2\end{array}\right)-1}$ given by the inverse image under the natural projection map.

We identify symmetric endomorphisms $R \in \operatorname{Sym}^{2}\left(\wedge^{2} \mathbb{R}^{n}\right)$ with their associated quadratic form $q_{R} \in \mathbb{R}\left[x_{i j}\right]_{2}$, which is a polynomial in the Plücker coordinates $x_{i j}$ :

$$
q_{R}\left(x_{i j}\right)=\left\langle R\left(\sum_{i<j} x_{i j} e_{i} \wedge e_{j}\right), \sum_{i<j} x_{i j} e_{i} \wedge e_{j}\right\rangle .
$$

To simplify notation, we use the same symbol $R$ for both of these objects, and henceforth identify $\operatorname{Sym}^{2}\left(\wedge^{2} \mathbb{R}^{n}\right)=\mathbb{R}\left[x_{i j}\right]_{2}$. Under this identification, the subspace $\wedge^{4} \mathbb{R}^{n} \subset \operatorname{Sym}^{2}\left(\wedge^{2} \mathbb{R}^{n}\right)$ corresponds to the degree 2 part $\left(I_{\mathrm{Gr}_{2}(n)}\right)_{2} \subset \mathbb{R}\left[x_{i j}\right]_{2}$ of the graded ideal $I_{\mathrm{Gr}_{2}(n)}$. In particular, its orthogonal complement $\operatorname{Sym}_{b}^{2}\left(\wedge^{2} \mathbb{R}^{n}\right)$ shall be identified with the quotient $\mathbb{R}\left[\mathrm{Gr}_{2}(n)\right]_{2}=\mathbb{R}\left[x_{i j}\right]_{2} /\left(I_{\mathrm{Gr}_{2}(n)}\right)_{2}$, as claimed in (1.5).

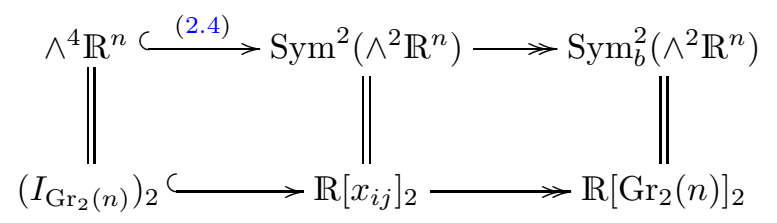


The sectional curvature function $\sec _{R}: \mathrm{Gr}_{2}^{+}\left(\mathbb{R}^{n}\right) \rightarrow \mathbb{R}$ determined by a (modified) curvature operator $R \in \mathbb{R}\left[x_{i j}\right]_{2}$ is its restriction to $\operatorname{Gr}_{2}^{+}\left(\mathbb{R}^{n}\right) \subset \wedge^{2} \mathbb{R}^{n}$, cf. (2.3). Since $\sec _{R}$ is invariant under the antipodal map on $S^{\left(\begin{array}{c}n \\ 2\end{array}\right)-1}$, it descends to a function on $\operatorname{Gr}_{2}\left(\mathbb{R}^{n}\right)$ also denoted by $\sec _{R}$.

Definition 2.1. Given $k \in \mathbb{R}$ and $n \geq 2$, let

$$
\Re_{\mathrm{sec} \geq k}(n)=\left\{R \in \operatorname{Sym}_{b}^{2}\left(\wedge^{2} \mathbb{R}^{n}\right): \sec _{R} \geq k\right\},
$$

and similarly for $\mathfrak{R}_{\mathrm{sec}>k}(n), \mathfrak{R}_{\mathrm{sec} \leq k}(n)$, and $\mathfrak{R}_{\mathrm{sec}<k}(n)$.

Remark 2.2. In order to simplify the exposition, we henceforth consider only the sectional curvature bound sec $\geq 0$, as other sectional curvature bounds can be easily recovered using the following elementary properties:

(i) $\mathfrak{R}_{\mathrm{sec} \geq k}(n)=\overline{\mathfrak{R}_{\mathrm{sec}>k}(n)}$ and $\mathfrak{R}_{\mathrm{sec} \leq k}(n)=\overline{\mathfrak{R}_{\mathrm{sec}<k}(n)}$;

(ii) $\operatorname{int}\left(\Re_{\mathrm{sec} \geq k}(n)\right)=\mathfrak{R}_{\mathrm{sec}>k}(n)$ and $\operatorname{int}\left(\mathfrak{R}_{\mathrm{sec} \leq k}(n)\right)=\mathfrak{R}_{\mathrm{sec}<k}(n)$;

(iii) $\sec _{R} \geq k$ if and only if $\sec _{R-k \text { Id }} \geq 0$, and $\sec _{R} \leq k$ if and only if $\sec _{k}$ Id $-R \geq 0$.

In particular, $\mathfrak{R}_{\mathrm{sec} \geq k}(n)$ and $\mathfrak{R}_{\mathrm{sec} \leq k}(n)$ are affine images of $\mathfrak{R}_{\mathrm{sec} \geq 0}(n)$.

The above are direct consequences of linearity of $R \mapsto \sec _{R}$ and $\sec _{\mathrm{Id}} \equiv 1$.

2.3. Discriminants. Given a polynomial $p(x)=a_{n} x^{n}+\cdots+a_{1} x+a_{0} \in \mathbb{C}[x]$, the discriminant of $p(x)$ is a polynomial $\operatorname{disc}_{x}(p(x)) \in \mathbb{Z}\left[a_{0}, \ldots, a_{n}\right]$ with integer coefficients whose variables are the coefficients of $p(x)$, defined as

$$
\operatorname{disc}_{x}(p(x))=a_{n}^{2 n-2} \prod_{i<j}\left(r_{i}-r_{j}\right)^{2},
$$

where $r_{1}, \ldots, r_{n} \in \mathbb{C}$ are the roots of $p(x)$. It can be computed explicitly in terms of $a_{i}, 0 \leq i \leq n$, by taking the determinant of the Sylvester matrix of $p(x)$ and $p^{\prime}(x)$. Clearly, $\operatorname{disc}_{x}(p(x))=0$ if and only if $p(x)$ has a root of multiplicity $\geq 2$. Note that $\operatorname{disc}_{x}(-p(x))=\operatorname{disc}_{x}(p(x))=\operatorname{disc}_{x}(p(-x))$. The discriminant of an $n \times n$ matrix $A$ is defined as the discriminant of its characteristic polynomial, that is, $\operatorname{disc}(A)=\operatorname{disc}_{x}(\operatorname{det}(A-x \operatorname{Id}))$. Thus, $\operatorname{disc}(A)=0$ if and only if $A$ has an eigenvalue of algebraic multiplicity $\geq 2$. For a description of $\operatorname{disc}(A)$ as a determinant, see [Par02]. Irreducibility of $\operatorname{disc}(A)$ for (symmetric) matrices is studied in Appendix B.

2.4. Spectrahedra and their shadows. We now recall basic notions from convex algebraic geometry, mostly without proofs. As a reference, we recommend [BPT13].

Definition 2.3. A spectrahedron is a set $S \subset \mathbb{R}^{n}$ of the form

$$
S=\left\{x \in \mathbb{R}^{n}: A+\sum_{i=1}^{n} x_{i} B_{i} \succeq 0\right\},
$$

where $A, B_{i} \in \operatorname{Sym}^{2}\left(\mathbb{R}^{d}\right)$ are symmetric matrices, and $M \succeq 0$ means $M$ is positivesemidefinite. A spectrahedral shadow is the image of a spectrahedron under a linear projection, i.e., a set $S \subset \mathbb{R}^{n}$ of the form

$$
S=\left\{x \in \mathbb{R}^{n}: \exists y \in \mathbb{R}^{m}, A+\sum_{i=1}^{n} x_{i} B_{i}+\sum_{j=1}^{m} y_{j} C_{j} \succeq 0\right\} .
$$

Remarks 2.4. The following are basic facts about spectrahedra and their shadows:

(i) Both spectrahedra and their shadows are convex semialgebraic sets. Furthermore, spectrahedra are closed; 
(ii) The class of spectrahedral shadows contains linear subspaces, polyhedra and all closed convex semialgebraic sets of dimension two [Sch18a];

(iii) The class of spectrahedral shadows is closed under intersections, linear projections, convex duality, (relative) interior, and closure.

The following notion was introduced by Helton and Vinnikov [HV07], and used in their proof of a conjecture of Peter Lax from 1958, see also [BPT13, Sec. 6.2.2].

Definition 2.5. A closed subset $S \subset \mathbb{R}^{n}$ is called an algebraic interior if it is the closure of a connected component of the set $\left\{x \in \mathbb{R}^{n}: p(x)>0\right\}$ for some polynomial $p \in \mathbb{R}\left[x_{1}, \ldots, x_{n}\right]$, which is called a defining polynomial of $S$.

Remark 2.6. Let $S$ be an algebraic interior, and $p$ be a defining polynomial of minimal degree. Then $p$ divides every defining polynomial of $S$, see [HV07, Lemma 2.1]. In particular, defining polynomials of minimal degree are unique up to a positive constant factor.

Lemma 2.7. Every spectrahedron $S$ with nonempty interior $\operatorname{int}(S)$ is an algebraic interior whose minimal defining polynomial $p$ satisfies $p(x) \neq 0$ for all $x \in \operatorname{int}(S)$.

Proof. Assume (without loss of generality) that $d$ in the semidefinite representation (2.7) of $S$ is minimal. We claim that $A+\sum_{i=1}^{n} x_{i} B_{i} \succ 0$ for all $x \in \operatorname{int}(S)$. Indeed, suppose that this does not hold at some $x_{*} \in \operatorname{int}(S)$. We may assume that $x_{*}=0$ and $e_{1} \in \operatorname{ker} A$. This implies that $A$ and $B_{i}$ are of the form

$$
A=\left(\begin{array}{cc}
0 & 0 \\
0 & A^{\prime}
\end{array}\right), \quad B_{i}=\left(\begin{array}{cc}
b_{i} & v_{i}^{\mathrm{t}} \\
v_{i} & B_{i}^{\prime}
\end{array}\right),
$$

where $A^{\prime}, B_{i}^{\prime} \in \operatorname{Sym}^{2}\left(\mathbb{R}^{d-1}\right), b_{i} \in \mathbb{R}$, and $v_{i} \in \mathbb{R}^{d-1}$ is a column vector. Since $0 \in \operatorname{int}(S)$, it follows that $\sum_{i=1}^{n} x_{i} b_{i} \geq 0$ for all $x \in \mathbb{R}^{n}$ near 0 , so $b_{i}=0,1 \leq i \leq n$. Then, applying the same reasoning to the appropriate $2 \times 2$-submatrices, it follows that $v_{i}=0,1 \leq i \leq n$. Therefore, $S$ admits the semidefinite representation $S=\left\{x \in \mathbb{R}^{n}: A^{\prime}+\sum_{i=1}^{n} x_{i} B_{i}^{\prime} \succeq 0\right\}$, contradicting the minimality of $d$.

The spectrahedron $S$ is hence an algebraic interior with defining polynomial $\operatorname{det}\left(A+\sum_{i=1}^{n} x_{i} B_{i}\right)$, which is positive in $\operatorname{int}(S)$. In particular, the minimal defining polynomial $p(x)$ of $S$ is also positive in $\operatorname{int}(S)$, see Remark 2.6.

Example 2.8. It is easy to show that the convex hull of two disjoint unit discs in the plane is not an algebraic interior (this set is called the football stadium). It is clearly a spectrahedral shadow, and not a spectrahedron by Lemma 2.7 .

Example 2.9. The $T V$ screen $\left\{(x, y) \in \mathbb{R}^{2}: x^{4}+y^{4} \leq 1\right\}$ is an algebraic interior and a spectrahedral shadow, but not a spectrahedron.

2.5. Quadratic forms on real projective varieties. Let $X \subset \mathbb{C} P^{N}$ be a real projective variety that is irreducible, not contained in any hyperplane, and whose set of real points $X(\mathbb{R}) \subset \mathbb{R} P^{N}$ is Zariski dense in $X$. Let $\mathbb{R}[X]$ be its homogeneous coordinate ring, i.e., the polynomial ring in $N+1$ variables modulo the homogeneous vanishing ideal of $X$. Inside the degree 2 part $\mathbb{R}[X]_{2}$, we consider the convex cone

$$
\Sigma_{X}=\left\{f \in \mathbb{R}[X]_{2}: f=g_{1}^{2}+\ldots+g_{s}^{2}, g_{i} \in \mathbb{R}[X]_{1}\right\}
$$

consisting of all sums of squares of linear forms, as well as the convex cone

$$
\mathrm{P}_{X}=\left\{f \in \mathbb{R}[X]_{2}: f \geq 0 \text { on } X(\mathbb{R})\right\}
$$


of all nonnegative quadratic forms on the real points $X(\mathbb{R})$. For the latter, note that the sign of $f$ at any point $x \in X(\mathbb{R})$ is well-defined because $f$ has even degree. Clearly, $\Sigma_{X} \subset \mathrm{P}_{X}$. Blekherman, Smith, and Velasco [BSV16, Thm. 1] showed that $\Sigma_{X}=\mathrm{P}_{X}$ if and only if $X$ has minimal degree, namely $\operatorname{deg}(X)=\operatorname{codim}(X)+1$. It is worth pointing out that $\mathrm{P}_{X}$ and $\Sigma_{X}$ are pointed closed convex cones with nonempty interior. Moreover, $\Sigma_{X}$ is a spectrahedral shadow: it is the image of the cone of positive-semidefinite matrices under the linear map sending a symmetric matrix $A$ to $x^{\mathrm{t}} A x \in \mathbb{R}[X]_{2}$ where $x=\left(x_{0}, \ldots, x_{N}\right)^{\mathrm{t}}$.

In the special case in which $X \subset \mathbb{C} P^{N}$ is a quadric, that is, the zero set of a single quadratic form, the above conclusion $\Sigma_{X}=\mathrm{P}_{X}$ holds without any additional assumptions and has been known for almost 100 years [Fin36].

Lemma 2.10 (Finsler). Let $A, B \in \operatorname{Sym}^{2}\left(\mathbb{R}^{d}\right)$. The following are equivalent:

(i) $\langle A v, v\rangle \geq 0$ for all $v \in \mathbb{R}^{d} \backslash\{0\}$ such that $\langle B v, v\rangle=0$;

(ii) there exists $x \in \mathbb{R}$ such that $A+x B \succeq 0$.

The analogous statement replacing all inequalities by strict inequalities also holds. Moreover, if (i) holds and there exists $v \in \mathbb{R}^{d} \backslash\{0\}$ with $\langle A v, v\rangle=\langle B v, v\rangle=0$ and $B$ has full rank, then $x$ in (ii) is unique.

Calabi [Cal64] independently found an elegant topological proof of this result; see also the survey [DP06] for other proofs and discussion.

Example 2.11. The sets $\mathrm{P}_{X}$ and $\Sigma_{X}$ have an important geometric interpretation when $X=\operatorname{Gr}_{2}(n)$ is the Grassmannian of 2-planes (2.5). Namely, keeping in mind the identifications described in Subsection 2.2, we have that

$$
\mathrm{P}_{\mathrm{Gr}_{2}(n)}=\mathfrak{R}_{\mathrm{sec} \geq 0}(n),
$$

and $\Sigma_{\mathrm{Gr}_{2}(n)}$ is the set of curvature operators with strongly nonnegative curvature, see [BM17, BM18].

In order to compare $\mathrm{P}_{\mathrm{Gr}_{2}(n)}$ and $\Sigma_{\mathrm{Gr}_{2}(n)}$ using the results in [BSV16], note that

$$
\operatorname{codim} \operatorname{Gr}_{2}(n)=\left(\begin{array}{l}
n \\
2
\end{array}\right)-1-2(n-2)=\frac{(n-2)(n-3)}{2},
$$

and, by [EH16, Ex. 4.38],

$$
\operatorname{deg} \operatorname{Gr}_{2}(n)=\frac{(2(n-2)) !}{(n-2) !(n-1) !} .
$$

Thus, $\operatorname{deg} \operatorname{Gr}_{2}(n)=$ codim $\mathrm{Gr}_{2}(n)+1$ if and only if $n \leq 4$. Therefore, by Blekherman, Smith, and Velasco [BSV16, Thm. 1], $\Sigma_{\mathrm{Gr}_{2}(n)}=\mathrm{P}_{\mathrm{Gr}_{2}(n)}$ if and only if $n \leq 4$. In particular, $\mathrm{P}_{\mathrm{Gr}_{2}(n)}$ is a spectrahedral shadow for $n \leq 4$. On the other hand, for all $n \geq 5$, there exists a nonnegative quadratic form $P \in \mathrm{P}_{\mathrm{Gr}_{2}(n)}$ that is not a sum of squares, which plays a crucial role in the proof of Theorem A (1). An explicit example of such $P \in \mathrm{P}_{\mathrm{Gr}_{2}(n)} \backslash \Sigma_{\mathrm{Gr}_{2}(n)}, n \geq 5$, found by Zoltek [Zol79], is given by: $R_{\text {Zol }}=x_{12}^{2}+2 x_{13}^{2}+2 x_{23}^{2}+2 x_{14}^{2}+x_{15}^{2}+x_{34}^{2}+2 x_{25}^{2}+2 x_{45}^{2}-2 x_{12} x_{34}-2 x_{12} x_{15}-2 x_{34} x_{15}$. Remark 2.12. Since real symmetric matrices are diagonalizable, $R \in \mathbb{R}\left[x_{i j}\right]_{2}$ is a sum of squares if and only if $R \succeq 0$, i.e.,

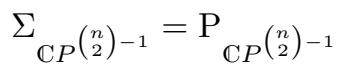

is the cone of positive-semidefinite curvature operators. 
2.6. Scheiderer's criterion. The only methods currently available to prove that a certain convex semialgebraic set is not a spectrahedral shadow were recently developed in a seminal work by Scheiderer [Sch18b]. For example, he showed that $\mathrm{P}_{X}$ is not a spectrahedral shadow when $X \subset \mathbb{C} P^{\left(\begin{array}{c}n+d \\ d\end{array}\right)-1}$ is the $d$ th Veronese embedding of $\mathbb{C} P^{n}$ for every $n \geq 2, d \geq 3$; or $n \geq 3, d \geq 2$. Note that these are exactly the cases in which the $d$ th Veronese embedding does not satisfy $\operatorname{deg}(X)=\operatorname{codim}(X)+1$, see [EH16, Sec. 2.1.2]

In order to present Scheiderer's criterion, recall the following basic definitions and facts of convex geometry. Given any subset $\Omega$ of a finite-dimensional real vector space $V$, the convex hull and conic hull of $\Omega$ are defined, respectively, as:

$$
\begin{aligned}
& \operatorname{conv}(\Omega)=\left\{\sum_{i=1}^{k} \alpha_{i} v_{i}: v_{i} \in \Omega, \alpha_{i} \geq 0, \sum_{i=1}^{k} \alpha_{i}=1, k \in \mathbb{N}\right\}, \\
& \operatorname{cone}(\Omega)=\left\{\sum_{i=1}^{k} \alpha_{i} v_{i}: v_{i} \in \Omega, \alpha_{i} \geq 0, k \in \mathbb{N}\right\} .
\end{aligned}
$$

Denote by $V^{\vee}$ the dual vector space of $V$. The cone dual of $\Omega$ is defined as

$$
\Omega^{*}=\left\{\lambda \in V^{\vee}: \lambda(x) \geq 0, \forall x \in \Omega\right\}
$$

and satisfies the following properties:

(i) $\Omega^{*} \subset V^{\vee}$ is a closed convex cone;

(ii) $\Omega^{*}=(\overline{\operatorname{conv}(\Omega)})^{*}$ and similarly for cone duals of any combinations of conic hull, convex hull, and closure;

(iii) $\Omega^{* *}=\left(\Omega^{*}\right)^{*}=\overline{\operatorname{cone}(\Omega)}$;

(iv) $\Omega^{*}$ is a spectrahedral shadow whenever $\Omega$ is a spectrahedral shadow.

For properties (iii) and (iv), see e.g. [BPT13, (5.11)] and [BPT13, Thm. 5.57], respectively.

Definition 2.13. Given $f \in \mathbb{R}\left[x_{1}, \ldots, x_{n}\right]$, its homogenization is the unique homogeneous polynomial $f^{h} \in \mathbb{R}\left[t, x_{1}, \ldots, x_{n}\right]$ with the same degree as $f$ such that $f^{h}\left(1, x_{1}, \ldots, x_{n}\right)=f\left(x_{1}, \ldots, x_{n}\right)$.

We are now in position to present the following convenient criterion to check if a semialgebraic set is not a spectrahedral shadow, extracted from Scheiderer [Sch18b].

Theorem 2.14. Let $L \subset \mathbb{R}\left[x_{1}, \ldots, x_{n}\right]$ be a finite-dimensional vector space with $1 \in L$, and let $f \in \mathbb{R}\left[x_{1}, \ldots, x_{n}\right]$ be a nonnegative polynomial which is not a sum of squares. Suppose that, for all $y \in \mathbb{R}^{n}$, the coefficients of $f^{h}\left(t, x_{1}-y_{1}, \ldots, x_{n}-y_{n}\right)$, considered as a polynomial in the new variable $t$, belong to $L$. Then the set

$$
K=\left\{g \in L: g(x) \geq 0 \text { for all } x \in \mathbb{R}^{n}\right\}
$$

is not a spectrahedral shadow.

Proof. For each $x \in \mathbb{R}^{n}$, let $\phi_{x} \in L^{\vee}$ be the evaluation functional $\phi_{x}(f)=f(x)$. Note that $K=\left\{\phi_{x}, x \in \mathbb{R}^{n}\right\}^{*}=C^{*}$, where

$$
C=\overline{\operatorname{conv}\left(\left\{\phi_{x}, x \in \mathbb{R}^{n}\right\}\right)} \subset L^{\vee} .
$$

Since $f$ is nonnegative but not a sum of squares, the same holds for its homogenization $f^{h}$. Using this and that the coefficients of $f^{h}\left(t, x_{1}-y_{1}, \ldots, x_{n}-y_{n}\right)$ belong to $L$, one can show, by the exact same reasoning as in [Sch18b, Ex. 4.20, 
Rem. 4.21], which follow from [Sch18b, Lem. 4.17, Prop. 4.18, Prop. 4.19], that $C$ is not a spectrahedral shadow.

Suppose, by contradiction, that $K$ is a spectrahedral shadow, so that its cone dual $K^{*}$ is also a spectrahedral shadow. By (iii), we have that $K^{*}=C^{* *}=\overline{\operatorname{cone}(C)}$. Since $1 \in L$, every evaluation $\phi_{x}$ is contained the affine hyperplane $H=\left\{\lambda \in L^{\vee}\right.$ : $\lambda(1)=1\}$ and hence $C \subset H$. In particular, $C=\overline{\operatorname{cone}(C)} \cap H=K^{*} \cap H$ is a spectrahedral shadow, providing the desired contradiction.

\section{Curvature operators in Dimension 4}

In this section, we prove Theorem $\mathrm{C}$ and statement (2) in Theorem A. For simplicity, we only treat the case sec $\geq 0$ for the reasons discussed in Remark 2.2. Furthermore, we denote by $\mathfrak{p}(R)$ the polynomial $\mathfrak{p}_{0}(R)$ from (1.3).

3.1. Curvature operators on 4-manifolds. Recall that the Hodge star operator is defined as $* \in \operatorname{Sym}^{2}\left(\wedge^{2} \mathbb{R}^{4}\right)$ corresponding to $\omega=e_{1} \wedge e_{2} \wedge e_{3} \wedge e_{4} \in \wedge^{4} \mathbb{R}^{4}$ under the identification (2.4). Its eigenvalues are \pm 1 and the corresponding eigenspaces $\wedge_{ \pm}^{2} \mathbb{R}^{4} \cong \mathbb{R}^{3}$ consist of so-called self-dual and anti-self-dual 2 -forms. Any symmetric endomorphism $R: \wedge^{2} \mathbb{R}^{4} \rightarrow \wedge^{2} \mathbb{R}^{4}$ can be represented by a block matrix with respect to the decomposition $\wedge^{2} \mathbb{R}^{4}=\wedge_{+}^{2} \mathbb{R}^{4} \oplus \wedge_{-}^{2} \mathbb{R}^{4} \cong \mathbb{R}^{6}$,

$$
R=\left(\begin{array}{cc}
A & B \\
B^{\mathrm{t}} & C
\end{array}\right)
$$

where $A$ and $C$ are symmetric $3 \times 3$-matrices and $B$ is any $3 \times 3$-matrix. Note that

$$
*=\left(\begin{array}{cc}
\mathrm{Id} & 0 \\
0 & -\mathrm{Id}
\end{array}\right),
$$

and the Bianchi identity (2.2) for $R$ as in (3.1) is $\operatorname{tr} A-\operatorname{tr} C=\operatorname{tr}(R *)=\langle R, *\rangle=0$. Finally, denote by $\operatorname{Sym}^{2}\left(\mathbb{C}^{6}\right)$ the space of complex symmetric $6 \times 6$-matrices.

The following characterization of $\Re_{\mathrm{sec} \geq 0}(4)$ was given by Thorpe [Tho72]. Note that this an immediate application of Finsler's Lemma 2.10.

Proposition 3.1 (Thorpe's trick). A curvature operator $R \in \operatorname{Sym}_{b}^{2}\left(\wedge^{2} \mathbb{R}^{4}\right)$ has $\sec _{R} \geq 0$ if and only if there exists $x \in \mathbb{R}$ such that $R+x * \succeq 0$, and analogously for $\sec _{R}>0$. Moreover, if $R$ has $\sec _{R} \geq 0$ but not $\sec _{R}>0$, then $x \in \mathbb{R}$ is unique.

3.2. Vanishing of $\mathfrak{p}$ on $\partial \mathfrak{R}_{\mathrm{sec} \geq 0}(4)$. We make repeated use of the complex matrix

$$
T=\operatorname{diag}(1,1,1, \sqrt{-1}, \sqrt{-1}, \sqrt{-1}),
$$

written in terms of the above identification (3.1). Note that $T$ is a square root of * and hence $\operatorname{det}(R+x *)=-\operatorname{det}(T R T+x \mathrm{Id})$, since $T(R+x *) T=T R T+x \mathrm{Id}$ and $\operatorname{det}\left(T^{2}\right)=-1$. Thus, $\operatorname{disc}_{x}(\operatorname{det}(R+x *))=\operatorname{disc}(T R T)$ by the properties of discriminants:

$$
\begin{aligned}
\operatorname{disc}_{x}(\operatorname{det}(R+x *)) & =\operatorname{disc}_{x}(-\operatorname{det}(T R T+x \mathrm{Id})) \\
& =\operatorname{disc}_{x}(\operatorname{det}(T R T-x \mathrm{Id})) \\
& =\operatorname{disc}(T R T) .
\end{aligned}
$$

Proposition 3.2. The polynomial $\mathfrak{p}(R)=\operatorname{disc}_{x}(\operatorname{det}(R+x *))$ vanishes on the (topological) boundary $\partial \mathfrak{R}_{\mathrm{sec} \geq 0}$. 
Proof. Let $R \in \partial \mathfrak{R}_{\mathrm{sec} \geq 0}$, which means that $\sec _{R} \geq 0$ but $R$ does not satisfy $\sec _{R}>$ 0 . By the Finsler-Thorpe trick (Proposition 3.1), there exists $x_{0} \in \mathbb{R}$ such that $R+x_{0} * \succeq 0$. It suffices to show $x_{0}$ is a root of $\operatorname{det}(R+x *)$ with multiplicity $\geq 2$.

Assume that $x_{0}$ is a simple root of this polynomial. Then $-x_{0}$ is an eigenvalue of $T R T$ with algebraic (and hence geometric) multiplicity 1 . Since $R+x_{0} *=$ $T^{-1}\left(T R T+x_{0} \mathrm{Id}\right) T^{-1}$, this implies that $\operatorname{dim} \operatorname{ker}\left(R+x_{0} *\right)=1$. As $R+x_{0} * \succeq 0$, it has 5 positive eigenvalues (counted with multiplicities). Since $x_{0}$ was assumed to be a simple root of $\operatorname{det}(R+x *)$, there exists $x$ near $x_{0}$ such that $\operatorname{det}(R+x *)>0$ and hence $R+x * \succ 0$, contradicting the fact that $R$ does not satisfy $\sec _{R}>0$.

Remark 3.3. The discriminant $\operatorname{disc}_{x}(\operatorname{det}(R+x *))$ is a homogeneous polynomial of degree 30 in the coefficients of $R$.

Remark 3.4. As a consequence of the above proof, $-x_{0}$ is an eigenvalue of $T R T$ with algebraic multiplicity $\geq 2$. We warn the reader that this does not imply that its geometric multiplicity is $\geq 2$, because $T R T$ is a complex symmetric matrix, hence not necessarily diagonalizable.

In fact, the geometric multiplicity of $-x_{0}$ cannot be always $\geq 2$. Indeed, on the one hand, $\operatorname{codim}\left(\partial \mathfrak{R}_{\mathrm{sec} \geq 0}\right)=1$. On the other hand, as in the proof of Proposition 3.5 below, since the set of symmetric $6 \times 6$-matrices with rank $\leq 4$ has codimension $\geq 3$ (see [HT84, p. 72]), it follows that

$$
\operatorname{codim}\left(\left\{R \in \operatorname{Sym}_{b}^{2}\left(\wedge^{2} \mathbb{R}^{4}\right): \exists x_{0} \in \mathbb{R}, \operatorname{rank}\left(R+x_{0} *\right) \leq 4\right\}\right) \geq 2 .
$$

3.3. Zeroes of $\mathfrak{p}$ in $\mathfrak{R}_{\mathrm{sec}>0}(4)$. We now study the interior vanishing locus of $\mathfrak{p}$.

Proposition 3.5. The zero set $\left\{R \in \mathfrak{R}_{\mathrm{sec}>0}(4): \mathfrak{p}(R)=0\right\}$ is a real subvariety of codimension $\geq 2$, hence its complement $\left\{R \in \mathfrak{R}_{\mathrm{sec}>0}(4): \mathfrak{p}(R)>0\right\}$ is connected.

Proof. Consider the orthogonal projection $\pi: \operatorname{Sym}^{2}\left(\wedge^{2} \mathbb{R}^{4}\right) \rightarrow \operatorname{Sym}_{b}^{2}\left(\wedge^{2} \mathbb{R}^{4}\right)$. Since the set of matrices of rank $\leq 4$ in $\operatorname{Sym}^{2}\left(\mathbb{C}^{6}\right)$ is a subvariety of codimension 3 , see e.g., [HT84, p. 72], the real subvariety $\mathcal{Y}=\left\{R \in \operatorname{Sym}^{2}\left(\wedge^{2} \mathbb{R}^{4}\right): \operatorname{rank}(R) \leq 4\right\}$ has codimension $\geq 3$. Thus, $\pi(\mathcal{Y})$ has codimension $\geq 2 \operatorname{since} \operatorname{dim} \pi(\mathcal{Y}) \leq \operatorname{dim} \mathcal{Y}$ and the ambient dimension drops by one. Thus, it suffices to show that for all $R \in \mathfrak{R}_{\sec >0}(4)$ with $\mathfrak{p}(R)=0$, there is $x_{0} \in \mathbb{R}$ with $\operatorname{rank}\left(R+x_{0} *\right) \leq 4$, i.e., $R \in \pi(\mathcal{Y})$.

Choose $\lambda \in \mathbb{R}$ such that $R+\lambda * \succ 0$ and an invertible real $6 \times 6$-matrix $S$ such that $R+\lambda *=S^{\mathrm{t}} S$. Since $\mathfrak{p}(R)=0$, the polynomial $q(t)=\operatorname{det}(R+(\lambda+t) *)$ has a root $t_{0} \neq 0$ of multiplicity $\geq 2$. We claim that $\operatorname{rank}\left(R+x_{0} *\right) \leq 4$ for $x_{0}=\lambda+t_{0}$. Note that

$$
\begin{aligned}
q(t) & =\operatorname{det}\left(S^{\mathrm{t}} S+t *\right) \\
& =(\operatorname{det} S)^{2} \operatorname{det}\left(\operatorname{Id}+t\left(S^{\mathrm{t}}\right)^{-1} * S^{-1}\right) \\
& =(\operatorname{det} S)^{2} t^{6} \operatorname{det}\left(\frac{1}{t} \mathrm{Id}+\left(S^{\mathrm{t}}\right)^{-1} * S^{-1}\right) \\
& =(\operatorname{det} S)^{2} \frac{1}{s^{6}} \operatorname{det}\left(\left(S^{\mathrm{t}}\right)^{-1} * S^{-1}-s \mathrm{Id}\right),
\end{aligned}
$$

where $s=-\frac{1}{t}$. Since $q\left(t_{0}\right)=0$ and $q^{\prime}\left(t_{0}\right)=0$, it follows that $-\frac{1}{t_{0}}$ is an eigenvalue of $\left(S^{\mathrm{t}}\right)^{-1} * S^{-1}$ with algebraic multiplicity $\geq 2$. As this is a real symmetric matrix, the geometric multiplicity of $-\frac{1}{t_{0}}$ is also $\geq 2$. Thus,

$$
\begin{aligned}
\operatorname{rank}\left(R+\left(\lambda+t_{0}\right) *\right) & =\operatorname{rank}\left(\frac{1}{t_{0}}(R+\lambda *)+*\right) \\
& =\operatorname{rank}\left(*+\frac{1}{t_{0}} S^{\mathrm{t}} S\right)
\end{aligned}
$$




$$
=\operatorname{rank}\left(\left(S^{\mathrm{t}}\right)^{-1} * S^{-1}+\frac{1}{t_{0}} \mathrm{Id}\right) \leq 4 .
$$

Remark 3.6. It follows from (3.4) that the polynomial $t \mapsto \operatorname{det}(R+t *)$ only has real roots if $R \in \mathfrak{R}_{\mathrm{sec} \geq 0}(4)$, since $\left(S^{\mathrm{t}}\right)^{-1} * S^{-1}$ is a real symmetric matrix. In particular, its discriminant $\mathfrak{p}(R)$ is nonnegative, see (2.6).

3.4. $\mathfrak{R}_{\mathrm{sec} \geq 0}(4)$ as an algebraic interior. We now prove Theorems $\mathrm{C}$ and $\mathrm{A}(2)$.

Proof of Theorem $C$. The zero set $\{\mathfrak{p}(R)=0\}$ contains the topological boundary of $\mathfrak{R}_{\mathrm{sec} \geq 0}(4)$ by Proposition 3.2 and has codimension $\geq 2$ in its interior by Proposition 3.5. By direct inspection, $\mathfrak{p}(R)>0$ at $R=\operatorname{diag}(1,2,3,4,5,6) \in \mathfrak{R}_{\mathrm{sec} \geq 0}(4)$. This implies that $\Re_{\mathrm{sec} \geq 0}(4)$ is an algebraic interior with defining polynomial $\mathfrak{p}(R)$, see Definition 2.5. We claim that this polynomial $\mathfrak{p}: \operatorname{Sym}_{b}^{2}\left(\wedge^{2} \mathbb{R}^{4}\right) \rightarrow \mathbb{R}$ is irreducible over $\mathbb{R}$, and hence it is a minimal defining polynomial, see Remark 2.6.

Denote by $\widetilde{\mathfrak{p}}: \operatorname{Sym}^{2}\left(\wedge^{2} \mathbb{R}^{4}\right) \rightarrow \mathbb{R}$ the polynomial $\widetilde{\mathfrak{p}}=\mathfrak{p} \circ \pi$, where $\pi$ is the orthogonal projection onto $\operatorname{Sym}_{b}^{2}\left(\wedge^{2} \mathbb{R}^{4}\right)$. Clearly, $\mathfrak{p}$ is irreducible if and only if $\tilde{\mathfrak{p}}$ is irreducible. On the other hand, $\widetilde{\mathfrak{p}}(R)$ is given by the same formula (1.3) as $\mathfrak{p}(R)$ since shifts in the variable $x$ do not change the discriminant, see (2.6). It suffices to show that the complexification $\widetilde{\mathfrak{p}}: \operatorname{Sym}^{2}\left(\mathbb{C}^{6}\right) \rightarrow \mathbb{C}$ of $\widetilde{\mathfrak{p}}$ is irreducible over $\mathbb{C}$. This is a consequence of Proposition B.1 in the Appendix, because $\widetilde{\mathfrak{p}}(R)$ is the discriminant of $T R T$ according to (3.3), and $R \mapsto T R T$ is a linear isomorphism of $\operatorname{Sym}^{2}\left(\mathbb{C}^{6}\right)$.

Finally, the last statement in Theorem $\mathrm{C}$ follows from the fact that the closure of any two connected components of $\{\mathfrak{p}(R)>0\}$ can only intersect at boundary points and $\operatorname{Id} \in \operatorname{int}\left(\mathfrak{R}_{\mathrm{sec} \geq 0}(4)\right)$.

Proof of Theorem A (2). The set $\mathfrak{R}_{\mathrm{sec} \geq 0}(4)$ is a spectrahedral shadow as a consequence of the Finsler-Thorpe trick (Proposition 3.1), see also Example 2.11. Furthermore, it is not a spectrahedron by Lemma 2.7, since $\mathfrak{p}$ is a minimal defining polynomial for $\mathfrak{R}_{\mathrm{sec}>0}(4)$ by Theorem $\mathrm{C}$, and $\mathfrak{p}$ vanishes at the interior point Id $\in \mathfrak{R}_{\sec >0}(4)$, since $\mathfrak{p}(\operatorname{Id})=\operatorname{disc}_{x}(\operatorname{det}(\operatorname{Id}+x *))=\operatorname{disc}_{x}\left((1+x)^{3}(1-x)^{3}\right)=0$.

\section{Curvature operators in dimensions $\geq 5$}

Using the notation from Section 2, consider the Plücker embedding of the Grassmannian $\mathrm{Gr}_{2}(5)$ in $\mathbb{C} P^{9}$, and recall that $\mathrm{P}_{\mathrm{Gr}_{2}(n)} \subset \mathbb{R}\left[\mathrm{Gr}_{2}(n)\right]_{2}$ is the subset of nonnegative quadratic forms on the real points of $\mathrm{Gr}_{2}(n)$, see (2.9). The main step in the proof of Theorem A (1) is the following application of Theorem 2.14.

Proposition 4.1. The closed convex cone $\mathrm{P}_{\mathrm{Gr}_{2}(5)}$ is not a spectrahedral shadow.

Proof. By [BSV16, Thm. 1] or [Zol79], see Example 2.11, there exists a polynomial $P \in \mathrm{P}_{\mathrm{Gr}_{2}(5)} \backslash \Sigma_{\mathrm{Gr}_{2}(5)}$. In other words, $P$ is a nonnegative quadratic form that is not a sum of squares modulo the vanishing ideal of $\mathrm{Gr}_{2}(5)$, i.e., the ideal generated by the Plücker relations. We consider the affine chart $U$ of $\operatorname{Gr}_{2}(5)$ defined by the Plücker coordinate $x_{15}$ being nonzero. Every point in $U$ is a 2-plane of the form

$$
\left(e_{1}+x_{25} e_{2}+x_{35} e_{3}+x_{45} e_{4}\right) \wedge\left(x_{12} e_{2}+x_{13} e_{3}+x_{14} e_{4}+e_{5}\right),
$$

that is, the row span of the matrix

$$
\Gamma=\left(\begin{array}{lllll}
1 & x_{25} & x_{35} & x_{45} & 0 \\
0 & x_{12} & x_{13} & x_{14} & 1
\end{array}\right) .
$$




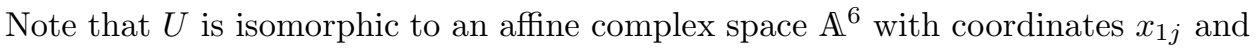

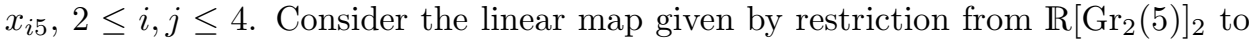

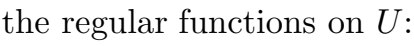

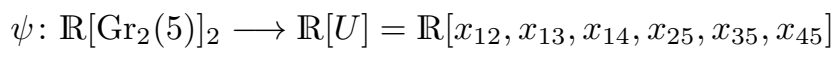

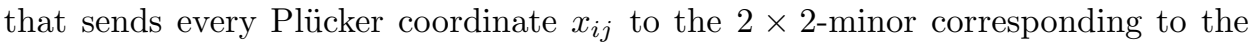

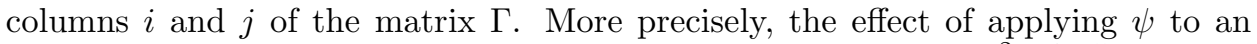

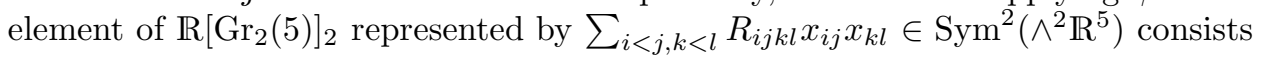

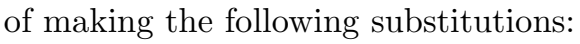

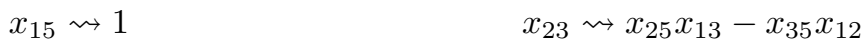

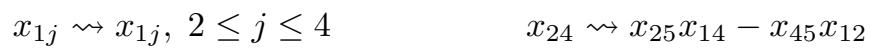

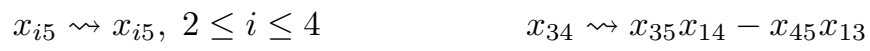

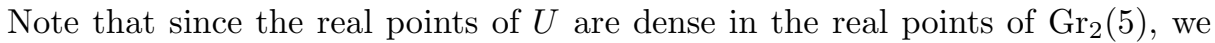

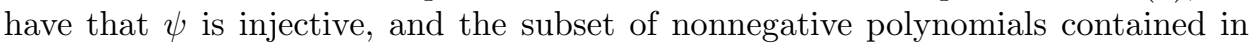

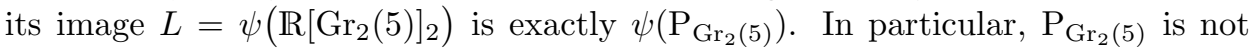

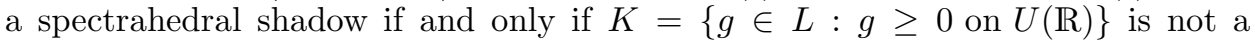

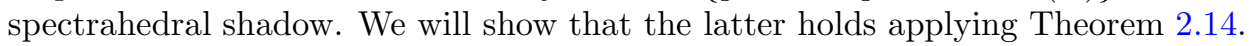

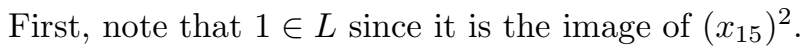

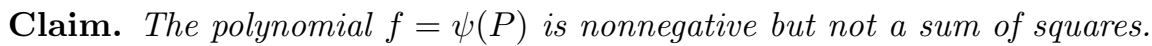

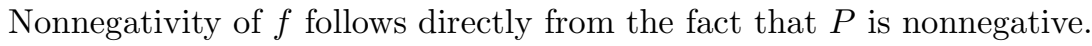

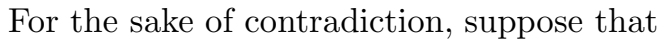

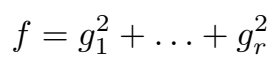

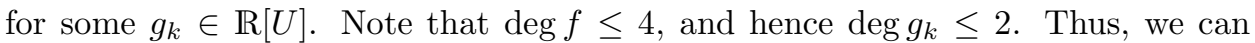

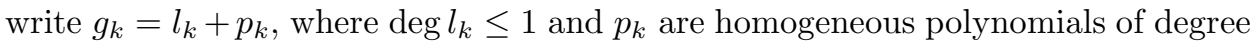

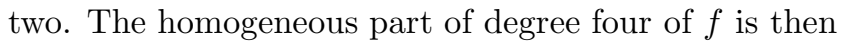

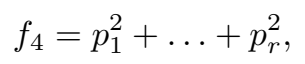

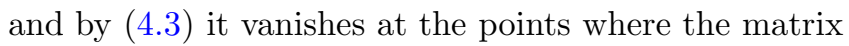

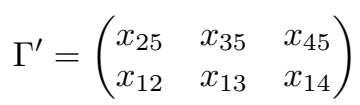

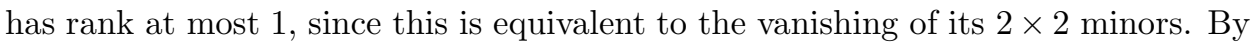

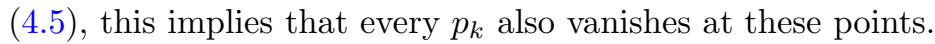

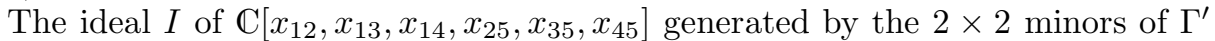

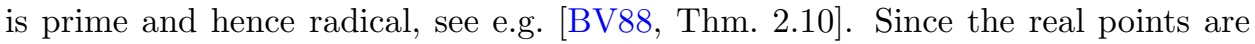

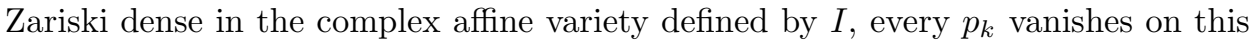

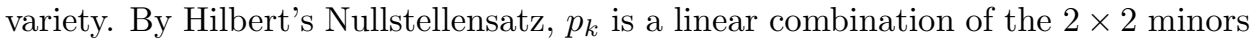

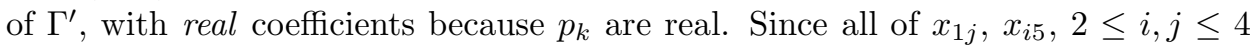

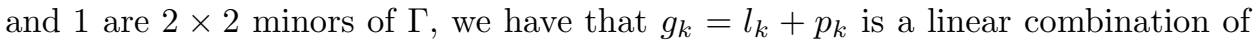

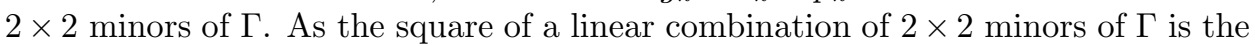

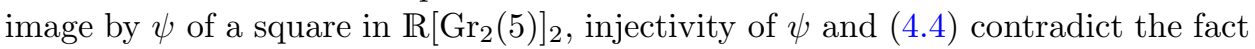

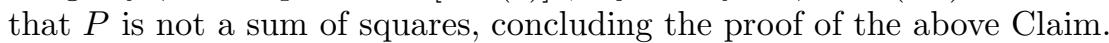

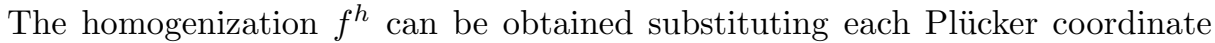

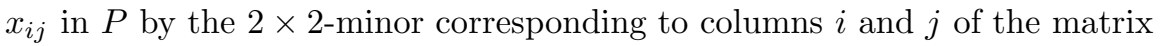

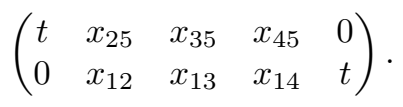


This and multilinearity of the determinant imply that all coefficients of

$$
f^{h}\left(t, x_{12}-y_{12}, \ldots, x_{45}-y_{45}\right),
$$

considered as a polynomial in $t$, belong to $L$ for all $y_{i j} \in \mathbb{R}$. Thus, by Theorem 2.14, we have that $\psi\left(\mathrm{P}_{\mathrm{Gr}_{2}(5)}\right)$, and hence $\mathrm{P}_{\mathrm{Gr}_{2}(5)}$, are not spectrahedral shadows.

Although our geometric applications only require the following result for $\mathrm{Gr}_{2}(n)$, $n \geq 5$, for the sake of completeness, we state and prove it in the more general case of the Grassmannian $\mathrm{Gr}_{k}(n)$ of $k$-planes in $n$-dimensional space.

Corollary 4.2. The closed convex cone $\mathrm{P}_{\mathrm{Gr}_{k}(n)}$ is not a spectrahedral shadow for all $n \geq 5$ and $2 \leq k \leq n-2$.

Proof. We proceed by induction on $n \geq 5$. For $n=5$, the conclusion holds by Proposition 4.1, since $\mathrm{Gr}_{2}(5) \cong \mathrm{Gr}_{3}(5)$ are naturally isomorphic.

For the induction step, fix $n \geq 5$ and suppose $\mathrm{P}_{\mathrm{Gr}_{k}(n)}$ is not a spectrahedral shadow for all $2 \leq k \leq n-2$. Since $\operatorname{Gr}_{k}(n+1) \cong \mathrm{Gr}_{n-k}(n+1)$ are naturally isomorphic, it suffices to show that $\mathrm{P}_{\mathrm{Gr}_{k}(n+1)}$ is not a spectrahedral shadow for all $2 \leq k \leq \frac{n+1}{2}$. Note that every such $k$ satisfies $2 \leq k \leq n-2$ because $n \geq 5$.

The cone on the Grassmannian of $k$-planes in $\mathbb{C}^{n}$,

$$
C \mathrm{Gr}_{k}(n)=\left\{v_{1} \wedge \cdots \wedge v_{k}: v_{i} \in \mathbb{C}^{n}\right\} \subset \wedge^{k} \mathbb{C}^{n},
$$

is an affine variety whose (affine) coordinate ring agrees with the homogeneous coordinate ring of $\operatorname{Gr}_{k}(n)$. The projection map $\pi: \mathbb{C}^{n+1} \rightarrow \mathbb{C}^{n}$ onto the first $n$ coordinates induces a linear map $\wedge^{k} \pi: \wedge^{k} \mathbb{C}^{n+1} \rightarrow \wedge^{k} \mathbb{C}^{n}$, which maps $C \operatorname{Gr}_{k}(n+1)$ onto $C \operatorname{Gr}_{k}(n)$. In particular, the associated homomorphism of coordinate rings given by composition with $\wedge^{k} \pi$ is injective. This implies that $\mathbb{R}\left[\operatorname{Gr}_{k}(n)\right]_{2}$ can be identified with a linear subspace of $\mathbb{R}\left[\operatorname{Gr}_{k}(n+1)\right]_{2}$. Note that $\mathrm{P}_{\mathrm{Gr}_{k}(n)} \subset \mathbb{R}\left[\mathrm{Gr}_{k}(n)\right]_{2}$ consists of the elements which are nonnegative on the real points $C \operatorname{Gr}_{k}(n)(\mathbb{R})$, and similarly for $\mathrm{P}_{\mathrm{Gr}_{k}(n+1)} \subset \mathbb{R}\left[\operatorname{Gr}_{k}(n+1)\right]_{2}$. Therefore,

$$
\mathrm{P}_{\mathrm{Gr}_{k}(n)}=\mathrm{P}_{\mathrm{Gr}_{k}(n+1)} \cap \mathbb{R}\left[\operatorname{Gr}_{k}(n)\right]_{2}
$$

because $\wedge^{k} \pi$ maps $C \operatorname{Gr}_{k}(n+1)(\mathbb{R})$ onto $C \operatorname{Gr}_{k}(n)(\mathbb{R})$ and pull-back of nonnegative functions are nonnegative. Since the intersection of a spectrahedral shadow with a linear subspace is also a spectrahedral shadow, it follows from the induction hypothesis and (4.8) that $\mathrm{P}_{\mathrm{Gr}_{k}(n+1)}$ is also not a spectrahedral shadow.

Proof of Theorem $A$ (1). We have that $\mathfrak{R}_{\mathrm{sec} \geq 0}(n)=\mathrm{P}_{\mathrm{Gr}_{2}(n)}$ by (2.10), and this is not a spectrahedral shadow by Corollary 4.2.

Remark 4.3. Both the cones from Corollary 4.2 and the counterexamples to the Helton-Nie conjecture by Scheiderer are dual cones to the convex hull of a highest weight orbit of some $\mathrm{SO}(n)$-representation, i.e., they are the dual cones to orbitopes [SSS11], see also [BB05]. For Scheiderer's examples these representations are $\operatorname{Sym}^{2 d}\left(\mathbb{R}^{n}\right), d \geq 2, n \geq 3$ and $(d, n) \neq(2,3)$, whereas here it is $\operatorname{Sym}^{2}\left(\wedge^{k} \mathbb{R}^{n}\right)$ for $n \geq 5$ and $2 \leq k \leq n-2$. Note that on the other hand for the representations $\operatorname{Sym}^{2}\left(\mathbb{R}^{n}\right), \operatorname{Sym}^{2 d}\left(\mathbb{R}^{2}\right)$ and $\wedge^{2} \mathbb{R}^{n}$ these cones are even spectrahedra [SSS11]. 


\section{Relaxations and Algorithms}

We now construct relaxations of $\mathfrak{R}_{\mathrm{sec} \geq 0}(n)$, that is, inner and outer approximations, proving Theorem B. These relaxations are then combined with semidefinite programming to establish simple algorithms to test whether a given curvature operator $R \in \operatorname{Sym}_{b}^{2}\left(\wedge^{2} \mathbb{R}^{n}\right)$ has $\sec _{R} \geq 0$, or any other sectional curvature bound, after a simple modification (Remark 2.2), see Algorithm 1. For $n=4$, we exploit the fact that $\mathfrak{R}_{\mathrm{sec}>0}(4)$ is a spectrahedral shadow to construct a more efficient algorithm based instead on Sturm's real root counting, see Algorithms 2 and 3.

5.1. Inner relaxations. Ideas similar to the Lasserre hierarchy [Las01] can be used to produce inner approximations of $\mathfrak{R}_{\mathrm{sec} \geq 0}(n)$ as follows.

Definition 5.1. Given $n \geq 2$ and a nonnegative integer $m \geq 0$, consider the linear map

$$
\varphi_{m}: \mathbb{R}\left[\operatorname{Gr}_{2}(n)\right]_{2} \longrightarrow \mathbb{R}\left[\operatorname{Gr}_{2}(n)\right]_{2 m+2}, \quad \varphi_{m}(P)=r^{m} \cdot P
$$

where $r=\sum_{i<j} x_{i j}^{2} \in \mathbb{R}\left[\operatorname{Gr}_{2}(n)\right]_{2}$ is the sum of squares of Plücker coordinates. Let $\mathfrak{I}_{m}$ be the preimage of the subset of sums of squares in $\mathbb{R}\left[\operatorname{Gr}_{2}(n)\right]_{2 m+2}$ under $\varphi_{m}$.

Clearly, every element of $\mathfrak{I}_{m}$ is a curvature operator $R$ with $\sec _{R} \geq 0$. The next result shows that, conversely, every curvature operator with $\sec _{R}>0$ is in some $\mathfrak{I}_{m}$. Observe that $\mathfrak{I}_{0}$ is precisely the set of curvature operators with strongly nonnegative curvature, see Example 2.11.

Proposition 5.2. For each $n \geq 2$, the collection $\mathfrak{I}_{m}, m \geq 0$, is a nested sequence of $\mathrm{O}(n)$-invariant spectrahedral shadows such that

$$
\mathfrak{R}_{\mathrm{sec}>0}(n) \subset \bigcup_{m \geq 0} \mathfrak{I}_{m} \subset \mathfrak{R}_{\mathrm{sec} \geq 0}(n) .
$$

In particular, we have $\overline{\bigcup_{m \geq 0} \mathfrak{I}_{m}}=\mathfrak{R}_{\mathrm{sec} \geq 0}(n)$.

Proof. First, observe that the subset of $\mathbb{R}\left[\operatorname{Gr}_{2}(n)\right]_{2 m+2}$ consisting of sums of squares is a spectrahedral shadow: it is the image of the cone of positive-semidefinite matrices under the linear map sending a symmetric matrix $A$ to

$$
[x]_{m+1}^{\mathrm{t}} A[x]_{m+1} \in \mathbb{R}\left[\operatorname{Gr}_{2}(n)\right]_{2 m+2},
$$

where $[x]_{m+1}$ denotes the column vector of all monomials of degree $m+1$. Thus, its preimage $\mathfrak{I}_{m}$ under the linear map $\varphi_{m}$ is a spectrahedral shadow. Furthermore, it is $\mathrm{O}(n)$-invariant because $r$ is fixed by the $\mathrm{O}(n)$-action. Since the product of two sums of squares is again a sum of squares, the sequence $\mathfrak{I}_{m}$ is nested.

Let $P \in \mathbb{R}\left[\mathrm{Gr}_{2}(n)\right]_{2}$ be a quadratic form that is positive on every real point of $\operatorname{Gr}_{2}(n)$, i.e., an element of $\mathfrak{R}_{\text {sec }>0}(n)$. We claim that $r^{m} \cdot P$ is a sum of squares of elements from $\mathbb{R}\left[\mathrm{Gr}_{2}(n)\right]_{m+1}$ for all sufficiently large $m$, i.e., $P \in \mathfrak{I}_{m}$. This follows from an appropriate Positivstellensatz, namely [Sch12, Cor. 4.2] applied to the pull-back $\mathcal{L}$ of the dual of the tautological line bundle on projective space via the Plücker embedding. Note that $\mathbb{R}\left[\operatorname{Gr}_{2}(n)\right]_{k}$ is the space of global sections of $\mathcal{L}^{\otimes k}$. The last statement then follows from Remark 2.2 (i).

Example 5.3. The curvature operator $R_{\text {Zol }}$ of Zoltek [Zol79] in Example 2.11 has sec $\geq 0$ but does not have strongly nonnegative curvature, i.e., lies in $\Re_{\text {sec } \geq 0}(5) \backslash \mathfrak{I}_{0}$. It can be checked that $R \in \mathfrak{I}_{1}$, using the package SOS [CKPP] for the computer algebra system Macaulay2 [GS]. 
Remark 5.4. For general $n$, both inclusions in Proposition 5.2 are strict. For example, since $\mathfrak{I}_{0}=\mathfrak{R}_{\mathrm{sec} \geq 0}(4)$, one has $\mathfrak{R}_{\mathrm{sec}>0}(4) \subsetneq \bigcup_{m \geq 0} \mathfrak{I}_{m}$ in this case. On the other hand, if we interpret $R_{\text {Zol }}$ from Example 2.11 as an element of $\mathbb{R}\left[\operatorname{Gr}_{2}(7)\right]_{2}$ instead of $\mathbb{R}\left[\mathrm{Gr}_{2}(5)\right]_{2}$, then $R_{\text {Zol }}$ is not contained in any $\mathfrak{I}_{m}$, and thus $\bigcup_{m \geq 0} \mathfrak{I}_{m} \subsetneq \mathfrak{R}_{\mathrm{sec} \geq 0}(7)$. This follows from the fact that $R_{\mathrm{Zol}}$ has a bad point in the sense of [Del97] at the point of $\mathrm{Gr}_{2}(7)$ corresponding to the 2-plane spanned by $e_{6}$ and $e_{7}$.

Remark 5.5. The curvature conditions corresponding to $\mathfrak{I}_{m}, m \geq 0$, are preserved under Riemannian submersions. More precisely, if $\pi:(\bar{M}, \bar{g}) \rightarrow(M, \mathrm{~g})$ is a Riemannian submersion and $R_{\bar{M}} \in \mathfrak{I}_{m}$, then also $R_{M} \in \mathfrak{I}_{m}$. This is a direct consequence of the Gray-O'Neill formula as presented in [BM18, Thm. B], which states:

$$
R_{M}=\left.\left(R_{\bar{M}}\right)\right|_{\wedge^{2} T M}+3 \alpha-3 \mathfrak{b}(\alpha),
$$

where $T M \subset T \bar{M}$ is the horizontal space, $\alpha$ is a quadratic form defined by the GrayO'Neill $A$-tensor, and $\mathfrak{b}: \operatorname{Sym}^{2}\left(\wedge^{2} T M\right) \rightarrow \wedge^{4} T M$ is the orthogonal projection. Thus, if $\varphi_{m}\left(R_{\bar{M}}\right) \in \mathbb{R}\left[\operatorname{Gr}_{2}(\bar{n})\right]_{2 m+2}$ is a sum of squares, then so is $\varphi_{m}\left(R_{M}\right) \in$ $\mathbb{R}\left[\operatorname{Gr}_{2}(n)\right]_{2 m+2}$, since $\varphi_{m}(3 \alpha)$ is also a sum of squares and $3 \mathfrak{b}(\alpha)$ is in the vanishing ideal of $\operatorname{Gr}_{2}(n)$.

5.2. Outer relaxations. We now observe that outer approximations of $\Re_{\sec \geq 0}(n)$ by spectrahedra can be constructed using [BM, Thm. A]. As usual, we identify traceless symmetric $p$-tensors $\psi \in \operatorname{Sym}_{0}^{p} \mathbb{R}^{n}$ with harmonic homogeneous polynomials $\psi \in \mathbb{R}\left[x_{1}, \ldots, x_{n}\right]_{p} \cap$ ker $\Delta$. The curvature term induced by $R \in \operatorname{Sym}_{b}^{2}\left(\wedge^{2} \mathbb{R}^{n}\right)$ in the Weitzenböck formula for traceless symmetric $p$-tensors is the symmetric linear endomorphism $\mathcal{K}\left(R, \operatorname{Sym}_{0}^{p} \mathbb{R}^{n}\right): \operatorname{Sym}_{0}^{p} \mathbb{R}^{n} \rightarrow \operatorname{Sym}_{0}^{p} \mathbb{R}^{n}$ determined by

$$
\left\langle\mathcal{K}\left(R, \operatorname{Sym}_{0}^{p} \mathbb{R}^{n}\right) \psi, \psi\right\rangle=c_{p, n} \int_{S^{n-1}}\langle R(x \wedge \nabla \psi(x)), x \wedge \nabla \psi(x)\rangle \mathrm{d} x,
$$

where $c_{p, n}>0$ is a constant, see [BM, Prop. 3.1].

Definition 5.6. Given $n \geq 2$ and a nonnegative integer $m \geq 0$, let

$$
\mathfrak{O}_{m}=\left\{R \in \operatorname{Sym}_{b}^{2}\left(\wedge^{2} \mathbb{R}^{n}\right): \mathcal{K}\left(R, \operatorname{Sym}_{0}^{p} \mathbb{R}^{n}\right) \succeq 0 \text { for all } 1 \leq p \leq m+1\right\} .
$$

Proposition 5.7. For each $n \geq 2$, the collection $\mathfrak{O}_{m}, m \geq 0$, is a nested sequence of $\mathrm{O}(n)$-invariant spectrahedra such that

$$
\bigcap_{m \geq 0} \mathfrak{O}_{m}=\mathfrak{R}_{\mathrm{sec} \geq 0}(n) .
$$

Proof. Since $\mathcal{K}\left(R, \operatorname{Sym}_{0}^{p} \mathbb{R}^{n}\right)$ depends linearly on $R$ and is $\mathrm{O}(n)$-equivariant, $\mathfrak{O}_{m}$ are (finite) intersections of $\mathrm{O}(n)$-invariant spectrahedra, hence $\mathrm{O}(n)$-invariant spectrahedra themselves. The inclusion $\mathfrak{R}_{\text {sec } \geq 0}(n) \subset \bigcap_{m \geq 0} \mathfrak{O}_{m}$ holds since the integrand in (5.1) is a sectional curvature; the reverse inclusion follows by [BM, Thm. A].

Remark 5.8. Note that the first step $\mathfrak{O}_{0}$ is precisely the set of curvature operators with nonnegative Ricci curvature [BM, Ex. 2.2]. Thus, in contrast with the inner approximations (see Remark 5.5), these curvature conditions are in general not preserved under Riemannian submersions, see e.g. [PW14].

Proof of Theorem B. Without loss of generality, we may consider only the case $k=0$, see Remark 2.2. The result now follows from Propositions 5.2 and 5.7. 
5.3. Algorithms to detect sec $\geq 0$. The relaxations constructed above enable the use of semidefinite programming to test membership in $\mathfrak{R}_{\mathrm{sec} \geq 0}(n)$. A semidefinite program optimizes a linear functional over a spectrahedral shadow $S$; in particular, it can be used to test whether $S$ is empty, and whether a given point belongs to $S$. Using interior-point methods, one can solve a semidefinite program up to a fixed precision in polynomial time in the size of the program description, see e.g. [NN94].

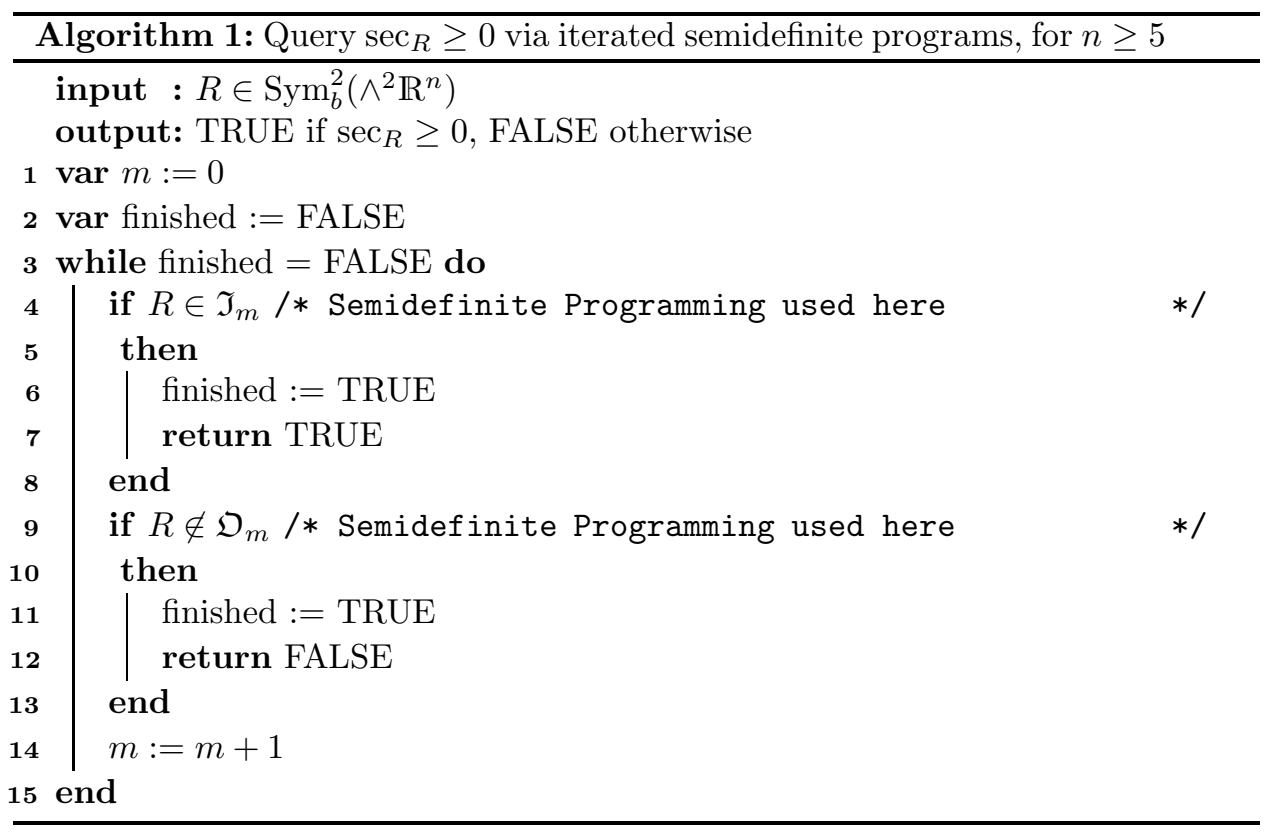

Proposition 5.9. For all $R \in \operatorname{Sym}_{b}^{2}\left(\wedge^{2} \mathbb{R}^{n}\right) \backslash \mathcal{B}$, where $\mathcal{B}:=\mathfrak{R}_{\mathrm{sec} \geq 0}(n) \backslash \bigcup_{m \geq 0} \mathfrak{I}_{m}$, Algorithm 1 terminates and returns TRUE if and only if $\sec _{R} \geq 0$. The set of bad inputs $\mathcal{B}$ has measure zero in $\operatorname{Sym}_{b}^{2}\left(\wedge^{2} \mathbb{R}^{n}\right)$ and is contained in $\partial \mathfrak{R}_{\mathrm{sec} \geq 0}(n)$.

Proof. These claims follow immediately from Propositions 5.2 and 5.7.

5.4. Efficient algorithms for $n=4$. Although semidefinite programming would not require iterations to detect membership in the spectrahedral shadow $\mathfrak{R}_{\mathrm{sec} \geq 0}(4)$, more efficient algorithms follow from the Finsler-Thorpe trick (Proposition 3.1).

Recall that the classical Sturm's algorithm returns the number of real roots (disregarding multiplicities) of a given univariate real polynomial $p \in \mathbb{R}[x]$ in any interval $[a, b]$ with $-\infty \leq a<b \leq+\infty$ and $p(a) \neq 0$ and $p(b) \neq 0$, see e.g. [BCR98, Cor. 1.2.10], or [BPR06, Sec. 2.2.2] for a more algorithmic viewpoint. This method produces partitions $-\infty=a_{1}<a_{2}<\cdots<a_{N}=+\infty$ which are root-isolating, that is, $p\left(a_{j}\right) \neq 0$ for all $j$ and $p(x)$ has exactly one root in $\left[a_{j}, a_{j+1}\right]$. In what follows, we convention that $p( \pm \infty)$ are interpreted as limits. Combining this procedure with Euclid's division algorithm (to compute greatest common divisors of polynomials), one can produce a common root-isolating partition for any finite collection of polynomials $p_{i} \in \mathbb{R}[x]$, i.e., $-\infty=a_{1}<a_{2}<\cdots<a_{N}=+\infty$ such that $p_{i}\left(a_{j}\right) \neq 0$ for all $i$ and $j$, and $\left[a_{j}, a_{j+1}\right]$ contains exactly one root of some $p_{i}(x)$. Note that if more than one $p_{i}(x)$ vanishes in $\left[a_{j}, a_{j+1}\right]$, then they must do so at the same point. 


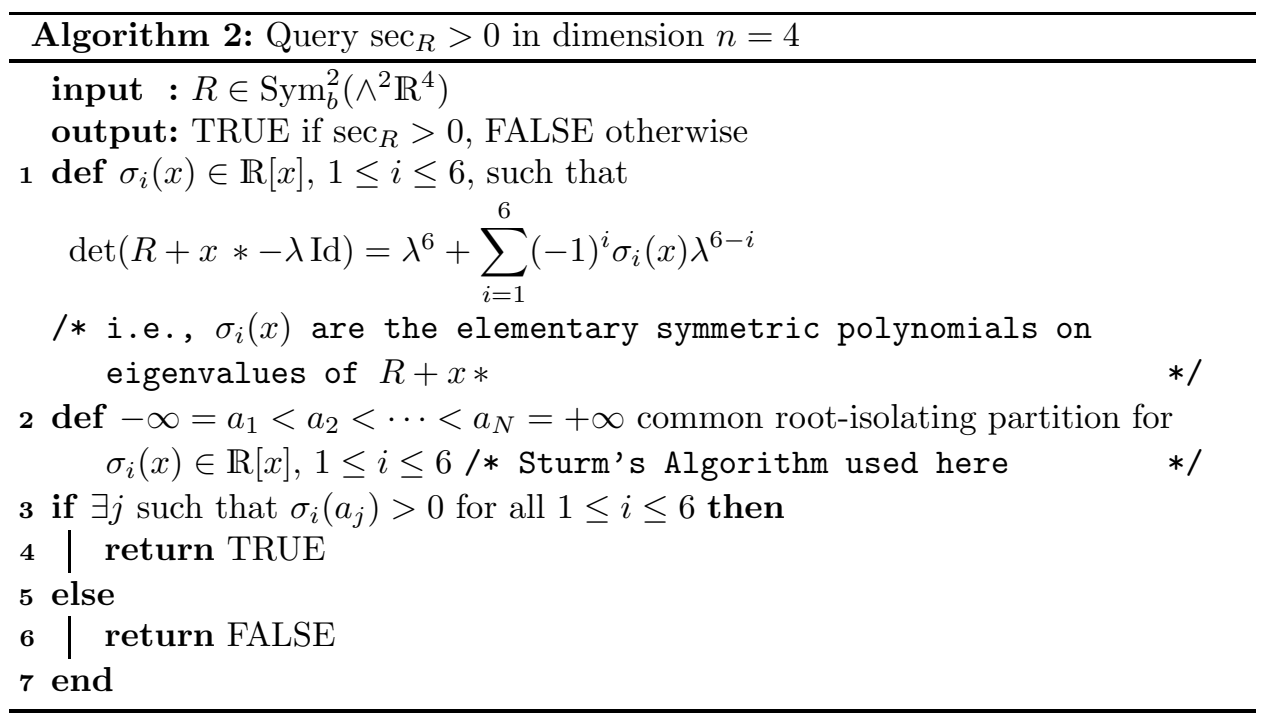

Proposition 5.10. For all $R \in \operatorname{Sym}_{b}^{2}\left(\wedge^{2} \mathbb{R}^{4}\right)$, Algorithm 2 terminates and returns TRUE if and only if $\sec _{R}>0$.

Proof. By the Finsler-Thorpe trick (Proposition 3.1), $\sec _{R}>0$ if and only if there exists $x \in \mathbb{R}$ such that $R+x \succ 0$, that is, the elementary symmetric polynomials $\sigma_{i}(x), 1 \leq i \leq 6$, in the eigenvalues of $R+x *$ are all positive.

Suppose the algorithm returns TRUE. Then there exists $1 \leq j \leq N$ such that $\sigma_{i}\left(a_{j}\right)>0$ for all $1 \leq i \leq 6$, so $R+a_{j} * \succ 0$ and hence $\sec _{R}>0$. Conversely, if $\sec _{R}>0$, let $x_{0} \in \mathbb{R}$ be such that $R+x_{0} * \succ 0$. Set $1 \leq j \leq N$ such that $x_{0} \in\left[a_{j}, a_{j+1}\right]$. If $x_{0}=a_{j}$ or $x_{0}=a_{j+1}$, then the algorithm clearly returns TRUE, so we may assume $x_{0} \in\left(a_{j}, a_{j+1}\right)$. Since $-\infty=a_{1}<a_{2}<\cdots<a_{N}=+\infty$ is a common root-isolating partition, one of the intervals $\left(a_{j}, x_{0}\right)$ or $\left(x_{0}, a_{j+1}\right)$ contains no roots of any $\sigma_{i}(x)$. Thus, either $\sigma_{i}\left(a_{j}\right)>0$ for all $1 \leq i \leq 6$, or $\sigma_{i}\left(a_{j+1}\right)>0$ for all $1 \leq i \leq 6$; which implies that the algorithm returns TRUE.

Proposition 5.11. For all $R \in \operatorname{Sym}_{b}^{2}\left(\wedge^{2} \mathbb{R}^{4}\right)$, Algorithm 3 terminates and returns TRUE if and only if $\sec _{R} \geq 0$.

Proof. Analogously to Proposition 5.10, $\sec _{R} \geq 0$ if and only if there exists $x \in \mathbb{R}$ such that $\sigma_{i}(x) \geq 0$ for all $1 \leq i \leq 6$.

Suppose the algorithm returns TRUE, so there exists $1 \leq j \leq N-1$ such that for all $1 \leq i \leq 6$ with $\sigma_{i}\left(a_{j}\right)<0$ and $\sigma_{i}\left(a_{j+1}\right)<0$, the polynomial $\sigma_{i}(x)$ has a root in $\left(a_{j}, a_{j+1}\right)$. Let $x_{0} \in\left(a_{j}, a_{j+1}\right)$ be the only root of some $\sigma_{i}(x)$ in that interval. We claim that $\sigma_{i}\left(x_{0}\right) \geq 0$ for all $1 \leq i \leq 6$, hence $\sec _{R} \geq 0$. This is shown by examining the signs of $\sigma_{i}\left(a_{j}\right)$ and $\sigma_{i}\left(a_{j+1}\right)$ and using that $-\infty=a_{1}<a_{2}<\cdots<a_{N}=+\infty$ is a common root-isolating partition for the $\sigma_{i}(x)$, as follows:

(1) If $\sigma_{i}\left(a_{j}\right)<0$ and $\sigma_{i}\left(a_{j+1}\right)<0$, then $\sigma_{i}\left(x_{0}\right)=0$ by the test in line $\mathbf{5}$;

(2) If $\sigma_{i}\left(a_{j}\right)>0$ and $\sigma_{i}\left(a_{j+1}\right)>0$, then clearly $\sigma_{i}\left(x_{0}\right) \geq 0$;

(3) If $\sigma_{i}\left(a_{j}\right)$ and $\sigma_{i}\left(a_{j+1}\right)$ have opposite signs, then $\sigma_{i}\left(x_{0}\right)=0$ by the Intermediate Value Theorem. 


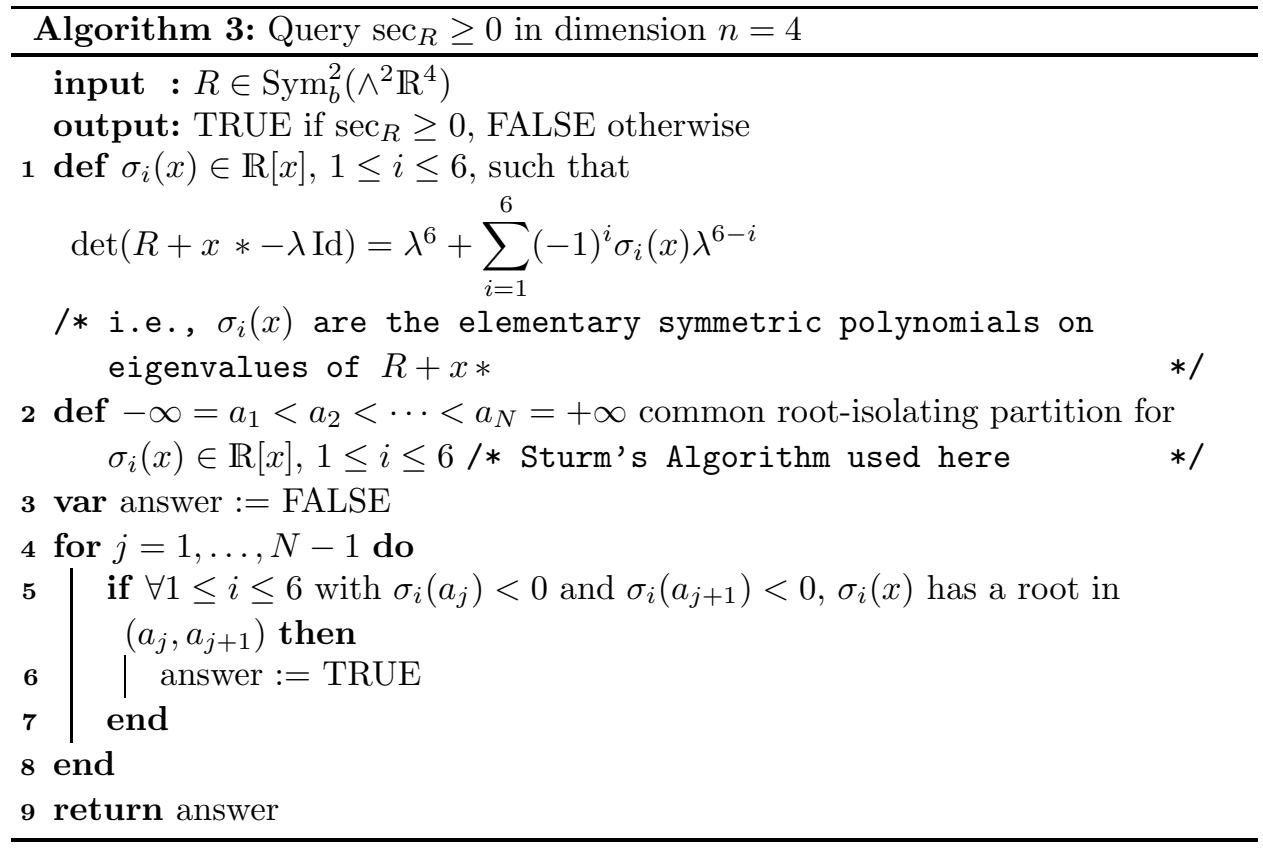

Conversely, if $\sec _{R} \geq 0$, choose $x_{0} \in \mathbb{R}$ such that $\sigma_{i}\left(x_{0}\right) \geq 0$ for all $1 \leq i \leq 6$. Let $1 \leq j \leq N-1$ be such that $x_{0} \in\left[a_{j}, a_{j+1}\right]$, and $1 \leq i \leq 6$ be such that $\sigma_{i}\left(a_{j}\right)<0$ and $\sigma_{i}\left(a_{j+1}\right)<0$. If $\sigma_{i}\left(x_{0}\right)>0$, then $\sigma_{i}(x)$ would have more than one root in $\left(a_{j}, a_{j+1}\right)$ contradicting the common root-isolating property, so $\sigma_{i}\left(x_{0}\right)=0$. Therefore, the algorithm returns TRUE.

\section{Appendix A. Curvature operators of Semi-Riemannian manifolds}

Recall that a semi-Riemannian (or pseudo-Riemannian) manifold $(M, \mathrm{~g}$ ) is a smooth manifold endowed with a semi-Riemannian metric g, i.e., a smooth section of the bundle $\operatorname{Sym}^{2}(T M)$ of symmetric bilinear forms that is nondegenerate (but possibly indefinite). As in the rest of the paper, since all our considerations are pointwise, given $p \in M$ we shall identify $T_{p} M \cong \mathbb{R}^{n}$, and consider

$$
\mathrm{g}(X, Y)=\langle G X, Y\rangle=-\sum_{i=1}^{\nu} x_{i} y_{i}+\sum_{j=\nu+1}^{n} x_{j} y_{j},
$$

where $\langle\cdot, \cdot\rangle$ is the standard inner product, $X=\left(x_{1}, \ldots, x_{n}\right), Y=\left(y_{1}, \ldots, y_{n}\right)$, and

$$
G=\operatorname{diag}(\underbrace{-1, \ldots,-1}_{\nu}, \underbrace{1, \ldots, 1}_{n-\nu}) .
$$

The integer $0 \leq \nu \leq n$ is called the signature of $\mathrm{g}$, and we henceforth assume $0<\nu<n$, i.e., $\pm \mathrm{g}$ are not Riemannian. Exactly as in the Riemannian case, semiRiemannian metrics g determine a (unique) Levi-Civita connection $\nabla$ on $T M$, see e.g. [O'N83, p. 11], and hence a curvature operator $R$, as in (2.1), that satisfies the first Bianchi identity (2.2). However, these equations must be interpreted appropriately: the standard inner product $\langle\cdot, \cdot\rangle$ on $\wedge^{2} T_{p} M \cong \wedge^{2} \mathbb{R}^{n}$ has to be replaced by 
the nondegenerate (but indefinite) symmetric bilinear form induced by g, namely

$$
\begin{aligned}
Q(X \wedge Y, Z \wedge W) & =\langle(G \wedge G)(X \wedge Y), Z \wedge W\rangle \\
& =\mathrm{g}(X, Z) \mathrm{g}(Y, W)-\mathrm{g}(X, W) \mathrm{g}(Y, Z),
\end{aligned}
$$

where $(G \wedge G)(X \wedge Y)=G X \wedge G Y$. Denote by $\operatorname{Sym}_{Q}^{2}\left(\wedge^{2} \mathbb{R}^{n}\right)$ the set of $Q$-symmetric $R: \wedge^{2} \mathbb{R}^{n} \rightarrow \wedge^{2} \mathbb{R}^{n}$, i.e., such that, for all $X \wedge Y, Z \wedge W \in \wedge^{2} \mathbb{R}^{n}$,

$$
Q(R(X \wedge Y), Z \wedge W)=Q(X \wedge Y, R(Z \wedge W)),
$$

and similarly for $\operatorname{Sym}_{Q, b}^{2}\left(\wedge^{2} \mathbb{R}^{n}\right)$ and (2.2). Decomposable elements $X \wedge Y \in \wedge^{2} \mathbb{R}^{n}$ are called nondegenerate if the restriction of $g$ to the 2-plane spanned by $X$ and $Y$ is nondegenerate, and degenerate otherwise. Note that $X \wedge Y$ is nondegenerate if and only if $Q(X \wedge Y, X \wedge Y) \neq 0$. Furthermore, $X \wedge Y$ is called definite if $Q(X \wedge Y, X \wedge Y)>0$, and indefinite if $Q(X \wedge Y, X \wedge Y)<0$; as these are equivalent to the restriction of $\mathrm{g}$ to $\operatorname{span}(X, Y)$ being definite, and indefinite, respectively.

The sectional curvature determined by $R \in \operatorname{Sym}_{Q}^{2}\left(\wedge^{2} \mathbb{R}^{n}\right)$ is

$$
\sec _{Q, R}(X \wedge Y)=\frac{Q(R(X \wedge Y), X \wedge Y)}{Q(X \wedge Y, X \wedge Y)},
$$

and is only defined for nondegenerate $X \wedge Y$, cf. (2.3). By a well-known result of Kulkarni, see [O'N83, p. 229], the only $R \in \operatorname{Sym}_{Q, b}^{2}\left(\wedge^{2} \mathbb{R}^{n}\right)$ with $\sec _{Q, R}(X \wedge Y) \geq 0$ for all nondegenerate $X \wedge Y$ is $R=0$. Furthermore, if the restriction of $\sec _{Q, R}$ to either definite or indefinite elements is bounded (from above and below), then $\sec _{Q, R}$ is constant everywhere. Thus, Definition 2.1 becomes vastly uninteresting.

Nevertheless, a suitable generalization of Definition 2.1 to the case of indefinite signature is given by the set $\Re_{\mathrm{sec} \geq k}(n, \nu)$ of $R \in \operatorname{Sym}_{Q, b}^{2}\left(\wedge^{2} \mathbb{R}^{n}\right)$ such that

$$
Q(R(X \wedge Y), X \wedge Y) \geq k Q(X \wedge Y, X \wedge Y), \quad \text { for all } X \wedge Y \in \wedge^{2} \mathbb{R}^{n} .
$$

Note that (A.1) is equivalent to $\sec _{Q, R} \geq k$ on definite elements and $\sec _{Q, R} \leq k$ on indefinite elements. It is easy to see that $\mathfrak{R}_{\mathrm{sec} \geq k}(n, \nu)=\mathfrak{R}_{\mathrm{sec} \geq k}(n)$ if $\nu=0$ or $\nu=n$, and $\mathfrak{R}_{\mathrm{sec} \geq k}(n, n-\nu)=\mathfrak{R}_{\mathrm{sec} \geq k}(n, \nu)$.

Arguably, condition (A.1) is not only algebraically, but also geometrically more natural than $\sec _{Q, R} \geq k$, since semi-Riemannian manifolds satisfy it pointwise if and only if they satisfy a local Alexandrov triangle comparison on the signed lengths of geodesics [AB08]. This curvature condition was first considered in [AH98], where comparison results for the Riccati equation are proven, and is also related to spacetime convex functions [AK17]. Our Theorem A carries over verbatim to this context:

Theorem A'. For all $k \in \mathbb{R}$ and $0 \leq \nu \leq n$, the set $\mathfrak{R}_{\mathrm{sec} \geq k}(n, \nu)$ is:

(1) not a spectrahedral shadow, if $n \geq 5$;

(2) a spectrahedral shadow, but not a spectrahedron, if $n=4$;

(3) a spectrahedron, if $n \leq 3$.

Proof. Consider the linear isomorphism $\psi_{Q}: \operatorname{Sym}_{Q}^{2}\left(\wedge^{2} \mathbb{R}^{n}\right) \rightarrow \operatorname{Sym}^{2}\left(\wedge^{2} \mathbb{R}^{n}\right)$, given by $\psi_{Q}(R)=(G \wedge G) \cdot R$. Note that it restricts to a linear isomorphism

$$
\psi_{Q}: \operatorname{Sym}_{Q, b}^{2}\left(\wedge^{2} \mathbb{R}^{n}\right) \longrightarrow \operatorname{Sym}_{b}^{2}\left(\wedge^{2} \mathbb{R}^{n}\right) \cong \mathbb{R}\left[\operatorname{Gr}_{2}(n)\right]_{2},
$$

and $\mathfrak{R}_{\mathrm{sec} \geq k}(n, \nu)=\psi_{Q}^{-1}\left(\mathfrak{R}_{\mathrm{sec} \geq k}(n)\right)$, so all conclusions follow from Theorem A. 
Moreover, preimages of the inner and outer approximations in Theorem B by $\psi_{Q}$ give analogous approximations of $\mathfrak{R}_{\mathrm{sec} \geq k}(n, \nu)$. However, these are no longer $\mathrm{O}(n)$-invariant, since $\psi_{Q}$ is not $\mathrm{O}(n)$-equivariant unless $\nu=0$ or $\nu=n$. Finally, Theorem $\mathrm{C}$ also carries over to $\mathfrak{R}_{\mathrm{sec} \geq k}(4, \nu)$, precomposing (1.3) with $\psi_{Q}$.

ApPendix B. IRREDUCIBILITy OF THE DISCRIMINANT OF SYMMETRIC MATRICES

In this Appendix, we study irreducibility of discriminants of symmetric matrices. Although the techniques are standard, we give complete proofs for the convenience of the reader, as the following does not seem to be easily available in the literature:

Proposition B.1. The discriminant of symmetric matrices disc: $\operatorname{Sym}^{2}\left(\mathbb{C}^{n}\right) \rightarrow \mathbb{C}$ is irreducible over $\mathbb{C}$ for all $n \geq 3$.

Remark B.2. The polynomial disc: $\operatorname{Sym}^{2}\left(\mathbb{C}^{n}\right) \rightarrow \mathbb{C}$ is a constant if $n=1$, and is not irreducible if $n=2$ since it is a product of two complex conjugate linear forms.

Consider the conjugation action on $\operatorname{Sym}^{2}\left(\mathbb{C}^{n}\right)$ of the (complex) Lie groups:

$$
\begin{aligned}
\mathrm{O}(n, \mathbb{C}) & =\left\{S \in \mathrm{GL}(n, \mathbb{C}): S^{\mathrm{t}} S=\mathrm{Id}\right\}, \\
\mathrm{SO}(n, \mathbb{C}) & =\{S \in \mathrm{O}(n, \mathbb{C}): \operatorname{det}(S)=1\} .
\end{aligned}
$$

Recall that $\operatorname{dim}_{\mathbb{C}} \mathrm{O}(n, \mathbb{C})=\operatorname{dim}_{\mathbb{C}} \mathrm{SO}(n, \mathbb{C})=\left(\begin{array}{l}n \\ 2\end{array}\right)$, and that $\mathrm{SO}(n, \mathbb{C})$ is an irreducible affine variety.

Lemma B.3. Let $A \in \operatorname{Sym}^{2}\left(\mathbb{C}^{n}\right)$ be a symmetric matrix whose $i$ th row has only zero entries, except possibly for its ith entry. Then the $\mathrm{O}(n, \mathbb{C})$-orbit of $A$ coincides with its $\mathrm{SO}(n, \mathbb{C})$-orbit.

Proof. Let $S \in \mathrm{O}(n, \mathbb{C}) \backslash \mathrm{SO}(n, \mathbb{C})$ be the reflection on the $i$ th coordinate, i.e., the diagonal matrix with entries 1 on the diagonal, except for a -1 at the $i$ th position. Clearly, $S$ is in the isotropy of $A$ and $\mathrm{O}(n, \mathbb{C})=\mathrm{SO}(n, \mathbb{C}) \cup(\mathrm{SO}(n, \mathbb{C}) \cdot S)$.

In the following, we describe the $\mathrm{O}(n, \mathbb{C})$-orbit of a symmetric matrix $A \in$ $\operatorname{Sym}^{2}\left(\mathbb{C}^{n}\right)$ by expressing it in terms of a convenient canonical form. To this end, following the notation of [Tho91], let $\Delta_{k}, \Lambda_{k} \in \operatorname{Sym}^{2}\left(\mathbb{C}^{k}\right)$ be the matrices given by

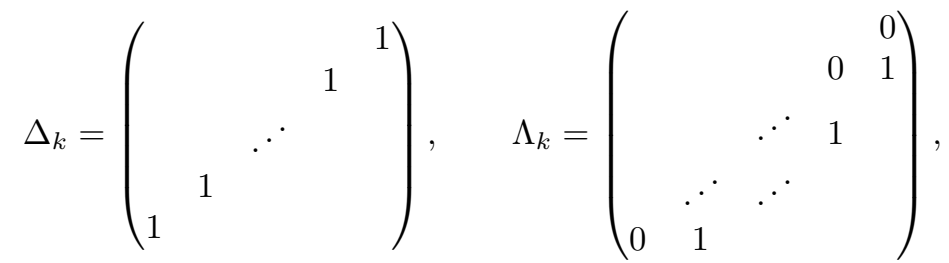

and fix $R_{k} \in \mathrm{GL}(k, \mathbb{C})$ such that $R_{k} \Delta_{k} R_{k}^{\mathrm{t}}=\mathrm{Id}_{k}$. Note that $\Lambda_{1}=0$. Furthermore, given $\lambda \in \mathbb{C}$, define $M_{k}(\lambda) \in \operatorname{Sym}^{2}\left(\mathbb{C}^{k}\right)$ by

$$
M_{k}(\lambda)=\lambda \operatorname{Id}_{k}+R_{k} \Lambda_{k} R_{k}^{\mathrm{t}} .
$$

Lemma B.4. Given $A \in \operatorname{Sym}^{2}\left(\mathbb{C}^{n}\right)$, there exist $\lambda_{1}, \ldots, \lambda_{\ell} \in \mathbb{C}$ eigenvalues of $A$, and $k_{1}, \ldots, k_{\ell} \in \mathbb{N}$, so that the $\mathrm{O}(n, \mathbb{C})$-orbit of $A$ contains the block diagonal matrix

$$
\left(\begin{array}{ccc}
M_{k_{1}}\left(\lambda_{1}\right) & & \\
& \ddots & \\
& & M_{k_{\ell}}\left(\lambda_{\ell}\right)
\end{array}\right) .
$$

Moreover, the characteristic polynomial of $A$ is $\operatorname{det}(A-t \mathrm{Id})=\prod_{i=1}^{\ell}\left(\lambda_{i}-t\right)^{k_{i}}$. 
Proof. We make use of the fact that one can bring pencils of symmetric matrices over $\mathbb{C}$ to a certain standard form. As proved e.g. in [Tho91, Sec. 5], there exist $\lambda_{1}, \ldots, \lambda_{\ell} \in \mathbb{C}$ eigenvalues of $A, k_{1}, \ldots, k_{\ell} \in \mathbb{N}$, and $S \in \mathrm{GL}(n, \mathbb{C})$ with the following property: for all $\rho \in \mathbb{C}$, the matrix $S\left(\rho \operatorname{Id}_{n}+A\right) S^{\mathrm{t}}$ is block diagonal with $\ell$ blocks of the form $\left(\rho+\lambda_{i}\right) \Delta_{k_{i}}+\Lambda_{k_{i}}$.

Letting $R \in \mathrm{GL}(n, \mathbb{C})$ be the block diagonal matrix with blocks $R_{k_{1}}, \ldots, R_{k_{\ell}}$, we have that $R S\left(\rho \operatorname{Id}_{n}+A\right) S^{\mathrm{t}} R^{\mathrm{t}}$ is a block diagonal matrix with blocks $M_{k_{i}}\left(\rho+\lambda_{i}\right)$. Since this holds for all $\rho \in \mathbb{C}$, it follows that $R S \in \mathrm{O}(n, \mathbb{C})$ and that $R S A(R S)^{\mathrm{t}}$ has the desired form (B.1).

The characteristic polynomial of $A$ is equal to that of (B.1), which is the product of the characteristic polynomials of its blocks $M_{k_{i}}\left(\lambda_{i}\right)$. These can be computed as:

$$
\begin{aligned}
\operatorname{det}\left(M_{k}(\lambda)-t \operatorname{Id}_{k}\right) & =\operatorname{det}\left(R_{k}\left(\Lambda_{k}+(\lambda-t) \Delta_{k}\right) R_{k}^{\mathrm{t}}\right) \\
& =\operatorname{det}\left(R_{k}\right)^{2} \operatorname{det}\left(\Lambda_{k}+(\lambda-t) \Delta_{k}\right) \\
& =(\lambda-t)^{k},
\end{aligned}
$$

because $\operatorname{det}\left(R_{k}\right)^{2}=(-1)^{\lfloor n / 2\rfloor}$ and $\operatorname{det}\left(\Lambda_{k}+(\lambda-t) \Delta_{k}\right)=(-1)^{\lfloor n / 2\rfloor}(\lambda-t)^{k}$.

We are now in the position to prove the main result of this Appendix:

Proof of Proposition B.1. The zero set $V$ of disc: $\operatorname{Sym}^{2}\left(\mathbb{C}^{n}\right) \rightarrow \mathbb{C}$ is a hypersurface in $\operatorname{Sym}^{2}\left(\mathbb{C}^{n}\right)$, and hence an equidimensional variety of (pure) codimension 1. Furthermore, since disc: $\operatorname{Sym}^{2}\left(\mathbb{C}^{n}\right) \rightarrow \mathbb{C}$ is $\mathrm{O}(n, \mathbb{C})$-invariant, by Lemma B.4 we have that $V$ is the union of the $\mathrm{O}(n, \mathbb{C})$-orbits of block diagonal matrices with blocks $M_{k_{1}}\left(\lambda_{1}\right), \ldots, M_{k_{\ell}}\left(\lambda_{\ell}\right)$ for all $\lambda_{i} \in \mathbb{C}$, and $k_{1}+\ldots+k_{\ell}=n$ with at least one $k_{i} \geq 2$, that is, $\ell \leq n-1$. For fixed $k_{1}, \ldots, k_{\ell}$, the set of such block matrices is parametrized by $\mathbb{C}^{\ell}$, and hence the union of the $\mathrm{O}(n, \mathbb{C})$-orbits of such matrices has dimension $\leq \ell+\operatorname{dim}_{\mathbb{C}} \mathrm{O}(n, \mathbb{C})$. If $\ell<n-1$, then $\ell+\operatorname{dim}_{\mathbb{C}} \mathrm{O}(n, \mathbb{C})<\operatorname{dim}_{\mathbb{C}} V$. Thus, since $V$ is equidimensional, it is the closure of the union of the $\mathrm{O}(n, \mathbb{C})$-orbits of all matrices

$$
M(\lambda)=\left(\begin{array}{llll}
M_{2}\left(\lambda_{1}\right) & & & \\
& \lambda_{2} & & \\
& & \ddots & \\
& & & \lambda_{n-1}
\end{array}\right)
$$

with $\lambda=\left(\lambda_{1}, \ldots, \lambda_{n-1}\right) \in \mathbb{C}^{n-1}$. Since $n \geq 3$, it suffices to take $\mathrm{SO}(n, \mathbb{C})$-orbits by Lemma B.3. In other words, $V$ is the closure of the image of the map

$$
\mathrm{SO}(n, \mathbb{C}) \times \mathbb{C}^{n-1} \longrightarrow \operatorname{Sym}^{2}\left(\mathbb{C}^{n}\right), \quad(S, \lambda) \mapsto S M(\lambda) S^{\mathrm{t}} .
$$

As the source is irreducible, the (closure of the) image is irreducible as well, which shows that $V$ is irreducible. Therefore, disc $=\phi^{m}$ for some irreducible polynomial $\phi: \operatorname{Sym}^{2}\left(\mathbb{C}^{n}\right) \rightarrow \mathbb{C}$ and $m \in \mathbb{N}$, so it remains to show that $m=1$. This can be seen, e.g., considering the restriction of disc: $\operatorname{Sym}^{2}\left(\mathbb{C}^{n}\right) \rightarrow \mathbb{C}$ to the curve

$$
\left(\begin{array}{ccccc}
1+x & \sqrt{-1} & & & \\
\sqrt{-1} & -1-x & & & \\
& & 1 & & \\
& & & \ddots & \\
& & & & n-2
\end{array}\right) \in \operatorname{Sym}^{2}\left(\mathbb{C}^{n}\right),
$$

which is a univariate polynomial in $x$ with a simple root at 0 , hence $m=1$. 


\section{REFERENCES}

[AB08] S. B. Alexander AND R. L. Bishop. Lorentz and semi-Riemannian spaces with Alexandrov curvature bounds. Comm. Anal. Geom., 16 (2008), 251-282.

[Afs11] B. AfSARI. Riemannian $L^{p}$ center of mass: existence, uniqueness, and convexity. Proc. Amer. Math. Soc., 139 (2011), 655-673.

[AH98] L. Andersson AND R. HowARD. Comparison and rigidity theorems in semiRiemannian geometry. Comm. Anal. Geom., 6 (1998), 819-877.

[AJLS17] N. Ay, J. Jost, H. V. LÊ, ANd L. Schwachhöfer. Information geometry, vol. 64 of Ergebnisse der Mathematik und ihrer Grenzgebiete. 3. Folge. A Series of Modern Surveys in Mathematics [Results in Mathematics and Related Areas. 3rd Series. A Series of Modern Surveys in Mathematics]. Springer, Cham, 2017.

[AK17] S. B. AlEXANDER AND W. A. KARR. Space-time convex functions and sectional curvature. In Lorentzian geometry and related topics, vol. 211 of Springer Proc. Math. Stat., 13-26. Springer, Cham, 2017.

[AL12] M. F. ANJos AND J. B. LASSERRE. Handbook on semidefinite, conic and polynomial optimization, vol. 166 of International Series in Operations Research \& Management Science. Springer, New York, 2012.

[Ama16] S.-I. AmARI. Information geometry and its applications, vol. 194 of Applied Mathematical Sciences. Springer, [Tokyo], 2016.

[Art27] E. ARTin. Über die Zerlegung definiter Funktionen in Quadrate. Abh. Math. Sem. Univ. Hamburg, 5 (1927), 100-115.

[BB05] A. Barvinok And G. Blekherman. Convex geometry of orbits. In Combinatorial and computational geometry, vol. 52 of Math. Sci. Res. Inst. Publ., 51-77. Cambridge Univ. Press, Cambridge, 2005.

[BCR98] J. Bochnak, M. Coste, And M.-F. Roy. Real Algebraic Geometry, vol. 36 of Ergebnisse der Mathematik und ihrer Grenzgebiete (3). Springer-Verlag, Berlin, 1998. Translated from the 1987 French original, Revised by the authors.

[BJV17] M. Banerjee, B. Jian, and B. C. Vemuri. Robust Fréchet Mean and PGA on Riemannian Manifolds with Applications to Neuroimaging. In M. Niethammer, M. Styner, S. Aylward, H. Zhu, I. Oguz, P.-T. Yap, and D. Shen, editors, Information Processing in Medical Imaging, 3-15, Cham, 2017. Springer International Publishing.

[BM] R. G. Bettiol And R. A. E. Mendes. Sectional curvature and Weitzenböck formulae. Indiana Univ. Math. J., to appear. arXiv:1708.09033.

[BM17] R. G. Bettiol And R. A. E. Mendes. Strongly nonnegative curvature. Math. Ann., 368 (2017), 971-986.

[BM18] R. G. Bettiol and R. A. E. Mendes. Strongly positive curvature. Ann. Global Anal. Geom., 53 (2018), 287-309.

[BPR06] S. Basu, R. Pollack, And M.-F. Roy. Algorithms in Real Algebraic Geometry, vol. 10 of Algorithms and Computation in Mathematics. Springer-Verlag, Berlin, second edition, 2006.

[BPT13] G. Blekherman, P. A. Parrilo, and R. R. Thomas. Semidefinite optimization and convex algebraic geometry, vol. 13 of MOS-SIAM Series on Optimization. Society for Industrial and Applied Mathematics (SIAM), Philadelphia, PA; Mathematical Optimization Society, Philadelphia, PA, 2013.

[BSV16] G. Blekherman, G. G. Smith, and M. Velasco. Sums of squares and varieties of minimal degree. J. Amer. Math. Soc., 29 (2016), 893-913.

[BV88] W. Bruns and U. Vetter. Determinantal rings, vol. 45 of Monografías de Matemática [Mathematical Monographs]. Instituto de Matemática Pura e Aplicada (IMPA), Rio de Janeiro, 1988.

[Cal64] E. Calabi. Linear systems of real quadratic forms. Proc. Amer. Math. Soc., 15 (1964), $844-846$.

[CKPP] D. Cifuentes, T. Kahle, P. A. Parrilo, and H. Peyrl. Sos: sums of squares. Version 2.0. A Macaulay2 package available at https://github.com/Macaulay2/M2/tree/master/M2/Macaulay2/packages .

[Del97] C. N. Delzell. Bad points for positive semidefinite polynomials. In Abstracts Amer. Math. Soc., vol. 18, 926-12, 1997. 
[DP06] K. Derinkuyu and M. C. Pinar. On the S-procedure and some variants. Math. Methods Oper. Res., 64 (2006), 55-77.

[EH16] D. EISENBUD AND J. HARRIS. 3264 and all that-a second course in algebraic geometry. Cambridge University Press, Cambridge, 2016.

[Faw19] H. FAwzI. The set of separable states has no finite semidefinite representation except in dimension $3 \times 2$. arXiv preprint arXiv:1905.02575, (2019).

[FCPS04] P. T. Fletcher, Conglin Lu, S. M. Pizer, and Sarang Joshi. Principal geodesic analysis for the study of nonlinear statistics of shape. IEEE Transactions on Medical Imaging, 23 (2004), 995-1005.

[Fin36] P. FinsleR. Über das Vorkommen definiter und semidefiniter Formen in Scharen quadratischer Formen. Comment. Math. Helv., 9 (1936), 188-192.

[Fod20] D. G. Fodor. Algebraic conditions for the positivity of sectional curvature. Beitr. Algebra Geom., 61 (2020), 283-295.

[Goe98] M. X. Goemans. Semidefinite programming and combinatorial optimization. In Proceedings of the International Congress of Mathematicians, Vol. III (Berlin, 1998), no. Extra Vol. III, 657-666, 1998.

[GS] D. R. Grayson and M. E. Stillman. Macaulay2, a software system for research in algebraic geometry. Available at https://faculty.math.illinois.edu/Macaulay2/.

[Hil88] D. HilberT. Ueber die Darstellung definiter Formen als Summe von Formenquadraten. Math. Ann., 32 (1888), 342-350.

[HN09] J. W. Helton And J. NiE. Sufficient and necessary conditions for semidefinite representability of convex hulls and sets. SIAM J. Optim., 20 (2009), 759-791.

[HT84] J. HARRIS AND L. W. TU. On symmetric and skew-symmetric determinantal varieties. Topology, 23 (1984), 71-84.

[HV07] J. W. HeLton AND V. Vinnikov. Linear matrix inequality representation of sets. Comm. Pure Appl. Math., 60 (2007), 654-674.

[Las01] J. B. LASSERRE. Global optimization with polynomials and the problem of moments. SIAM J. Optim., 11 (2000/01), 796-817.

[Nem07] A. Nemirovski. Advances in convex optimization: conic programming. In International Congress of Mathematicians. Vol. I, 413-444. Eur. Math. Soc., Zürich, 2007.

[NN94] Y. NeSTEROV AND A. NemirovskiI. Interior-point polynomial algorithms in convex programming, vol. 13 of SIAM Studies in Applied Mathematics. Society for Industrial and Applied Mathematics (SIAM), Philadelphia, PA, 1994.

[O'N83] B. O'NeILl. Semi-Riemannian geometry, vol. 103 of Pure and Applied Mathematics. Academic Press, Inc. [Harcourt Brace Jovanovich, Publishers], New York, 1983. With applications to relativity.

[Par00] P. A. PARRILO. Structured semidefinite programs and semialgebraic geometry methods in robustness and optimization. PhD thesis, California Institute of Technology, 2000.

[Par02] B. N. PARlett. The (matrix) discriminant as a determinant. Linear Algebra Appl., 355 (2002), 85-101.

[PS03] P. A. Parrilo and B. Sturmfels. Minimizing polynomial functions. In Algorithmic and quantitative real algebraic geometry (Piscataway, NJ, 2001), vol. 60 of DIMACS Ser. Discrete Math. Theoret. Comput. Sci., 83-99. Amer. Math. Soc., Providence, RI, 2003.

[PW14] C. Pro and F. Wilhelm. Riemannian submersions need not preserve positive Ricci curvature. Proc. Amer. Math. Soc., 142 (2014), 2529-2535.

[Rag08] P. RAGHAVENDRA. Optimal algorithms and inapproximability results for every CSP? [extended abstract]. In STOC'08, 245-254. ACM, New York, 2008.

[Sch12] C. Scheiderer. A Positivstellensatz for projective real varieties. Manuscripta Math., 138 (2012), 73-88.

[Sch18a] C. Scheiderer. Semidefinite representation for convex hulls of real algebraic curves. SIAM J. Appl. Algebra Geom., 2 (2018), 1-25.

[Sch18b] C. Scheiderer. Spectrahedral shadows. SIAM J. Appl. Algebra Geom., 2 (2018), $26-44$.

[SSS11] R. Sanyal, F. Sottile, And B. Sturmfels. Orbitopes. Mathematika, 57 (2011), 275314 .

[Tho72] J. A. Thorpe. On the curvature tensor of a positively curved 4-manifold. In Proceedings of the 13th Biennial Seminar of the Canadian Mathematical Congress (Dalhousie 
Univ., Halifax, N.S., 1971), Vol. 2, 156-159. Canad. Math. Congr., Montreal, Que., 1972.

[Tho91] R. C. Thompson. Pencils of complex and real symmetric and skew matrices. Linear Algebra Appl., 147 (1991), 323-371.

[Wei72] A. Weinstein. Remarks on curvature and the Euler integrand. J. Differential Geometry, $6(1971 / 72), 259-262$.

[Zol79] S. M. ZoLTEK. Nonnegative curvature operators: some nontrivial examples. J. Differential Geom., 14 (1979), 303-315.

City University of New York (Lehman College)

Department of Mathematics

250 Bedford PARK Blvd W

Bronx, NY, 10468, USA

Email address: r.bettiol@lehman.cuny.edu

Technische Universität Dresden

Institut FÜR GeOMETRIE

Willersbau, WIL B 109

ZELLESCHER Weg 12-14

01069 Dresden, Germany

Email address: mario.kummer@tu-dresden.de

UNIVERSITY OF OKLAHOMA

Department of Mathematics

601 Elm Ave

Norman, OK, 73019-3103, USA

Email address: ricardo.mendes@ou.edu 